\title{
Trichopteran fauna of a hydrographic basin with a complex geological history (Umbria - Central Italy)
}

\author{
C. Corallini ${ }^{1 *}$, F. Cianficconi ${ }^{1}$ and B. Todini ${ }^{2}$ \\ 1 Department of Chemistry, Biology, Biotechnology, University of Perugia 06123, Perugia, Italy \\ 2 ARPA Umbria (Regional Environmental Protection Agency in Umbria), Via Pievaiola S. Sisto, 06132 - Perugia, Italy
}

Received 3 December 2014; Accepted 4 March 2015

\begin{abstract}
We set the first comprehensive inventory of Trichoptera species from the Topino River basin (Umbria, Italy), based on research carried out from 1947 to 2006 at 61 lotic sites (including rheocrenous and rheolimnocrenous springs and watercourses) and 1 lentic site (marsh). In total, 78 species and 5 subspecies from 17 families were collected. The species belong to several chorological categories. The inventory comprises 16 species and 5 subspecies endemic to the Italian fauna with Rhyacophila italica, Drusus camerinus, Drusus improvisus and Allogamus ausoniae limited to the central Apennines. The presence of Beraeodes minutus and Erotesis baltica in the Fonti del Clitunno and Wormaldia pulla marlieri in the Fergia River is noteworthy because they are rare species in Italy. Almost 60 years of research now allow us to draw a synthesis of Trichoptera diversity and distribution in light of the geological history at the drainage basin level.
\end{abstract}

Key words: Trichoptera / Topino basin / tectonics

\section{Introduction}

Research carried out from 1947 (Moretti, 1949) to 2005 (Cianficconi et al., 2007; Todini et al., 2009) on the Trichoptera fauna of the ten drainage basins of Umbria, Central Italy, allowed us to identify numerous lotic and lentic water bodies, and a variety of rheocrenous and limnocrenous springs in the Topino River basin (Fig. 1). From 1947 to 1953, further investigations were conducted by G.P. Moretti (Istituto di Zoologia dell'Università di Camerino) in the springs of the Topino River (Moretti, 1950, 1952; Scaramucci, 1948-49; Gianformaggio, 194950; Penna Rocchigiani, 1949-50), in the lake dell'Aiso (Moretti, 1952; Morroni, 1948-49; Ravagli, 1952-53), in the marsh di Colfiorito (Moretti, 1952) and in the spring Fonti del Clitunno (Moretti, 1949, 1952). Subsequently (1968-1996), research on the Trichoptera of the Topino basin was continued by G.P. Moretti and collaborators of the former Istituto di Zoologia dell'Università di Perugia and by collector Piero Salerno (Cianficconi et al., 1994, 2000, 2006; Moretti et al., 2006). The investigations were continued at sites previously studied (Di Giovanni et al., 1992; Cianficconi et al., 1998) and extended to other localities along the Topino River (Lipparelli, 1978-79; Montanucci, 1994-95; Cappelletti, 1997-98) and its tributaries, namely Rio Fergia (Farano, 1996-97),

\footnotetext{
*Corresponding author: drusinae@libero.it
}

Caldognola stream (Brunetti, 1994-95), Menotre River (Guidi, 1971-72; Fortini, 1987-88; Ricciolini, 2000-01; Todini, 2005), Chiona stream (Fucchi, 1995-96), Marroggia stream (Arena, 1990-91), the springs of Monte Subasio (Romozzi, 1989-90; Cardinali, 1992-93; Cianficconi et al., 2006) and Fonti del Clitunno (Corvelli, 1972-73). Almost 60 years of research now allow us to draw a synthesis of Trichoptera diversity and distribution in light of the geological history at the drainage basin level.

\section{Study area}

The hydrographic basin of the Topino $\left(1231 \mathrm{~km}^{2}\right)$, in the central-eastern area of Umbria, Italy (Fig. 1), is characterized by numerous lotic ecosystems, including rheocrenous and limnocrenous springs (Fonti del Clitunno), running and still waters (Palude di Colfiorito). Many springs have been greatly modified to provide drinking water for humans and animals. Lago dell' Aiso is a small lake fed by artesian springs. The Topino River is $48 \mathrm{~km}$ long. It originates at $670 \mathrm{~m}$ a.s.1. on the eastern slopes of Mt. Pennino in the Bagnara springs. It flows on a calcareous substrate in a narrow valley between Mt. Pennino in the east, and Mt. Subasio in the west. The Topino River flows into the Chiascio River (at $174 \mathrm{~m}$ a.s.1.), which is a tributary of the Tiber River. The Topino 


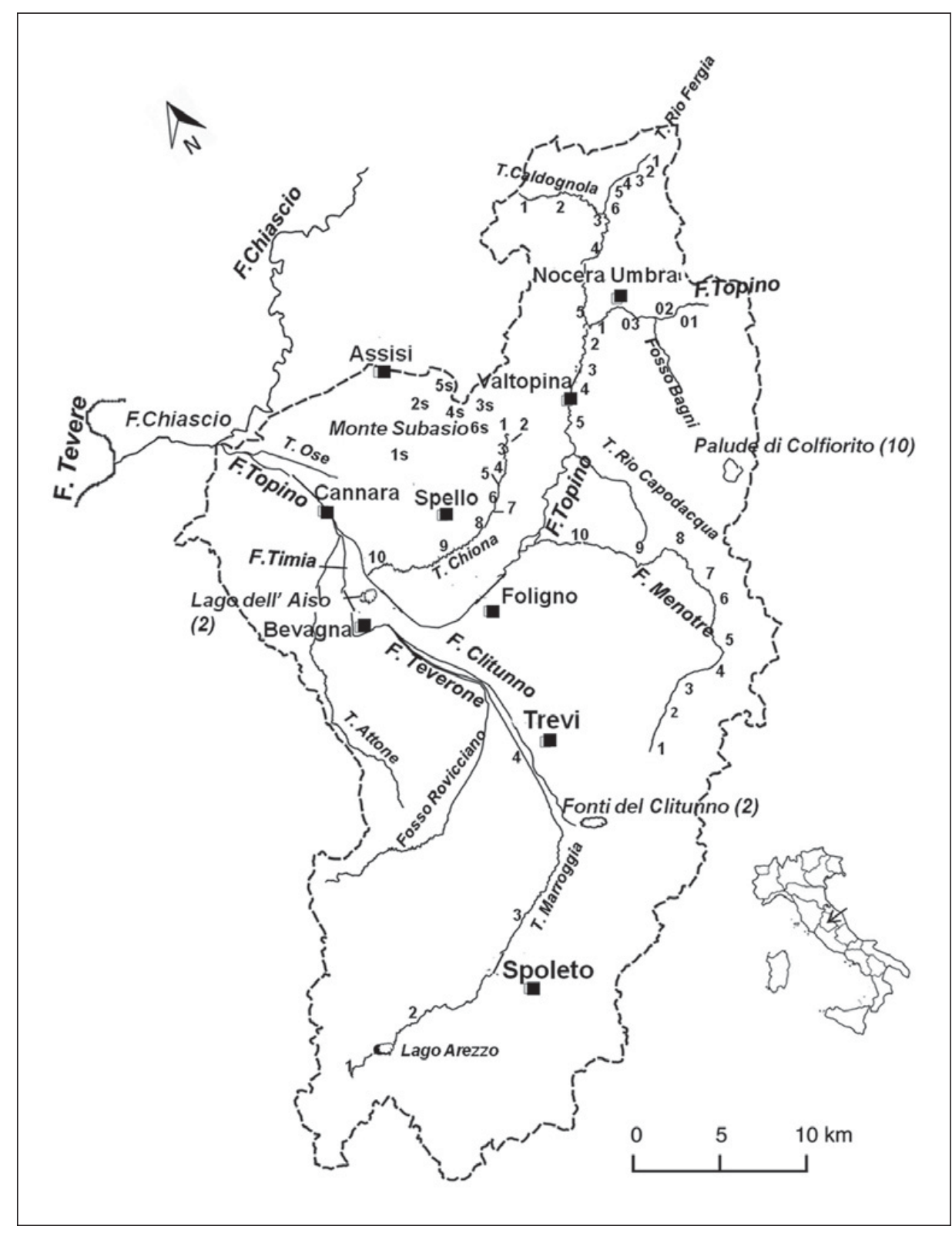

Fig. 1. Map of the Topino basin with sampling sites indicated by numbers. For Colfiorito, Aiso and Clitunno the total number of sampling sites are indicated in brackets; s, fonti (spring); F., river; T., stream; fosso, rio, watercourses; Palude, marsh; full squares, towns and villages.

also has several tributaries, namely Caldognola stream and Chiona stream on the right bank, and Menotre River, Marroggia stream, Rio di Capodacqua and Timia River on the left bank.

The Topino River basin is located on a geological area characterized by Mesozoic and Cenozoic marine strata of the Umbria-Marche Apennines and by continental deposits dating from the Pliocene up to the Holocene. The complex tectonic phenomena in central Italy from 5.33 million years ago (Miocene-Pliocene boundary) played a fundamental role in the geology and the structure of the Topino River basin (Colacicchi and Bizzarri, 2008; Colacicchi, 2010). At the beginning of the Pliocene, tectonic tension which already caused the rise of the
Tuscan mountains, moved towards the Umbria-Marche zone forming further structures and ridges. The location of the latter delineated a large depression with two branches (nowadays named Valle Tiberina to the west and Valle Umbra to the east), also referred to as Lacus Tiberinus (Fig. 2). In the classical scenario, this great lake supposedly had the shape of an upturned $\mathrm{Y}$ and a surface area of about $150 \mathrm{~km}^{2}$ (Lotti, 1917; Desplanques, 1969). Rainwaters were drained into this lake and, since there was no outlet, gradually filled both branches. Initially the basin was characterized by a fluvial-lacustrine regime that, in a short time, evolved towards a true lake environment which lasted about 2 million years in the Late Pliocene. 


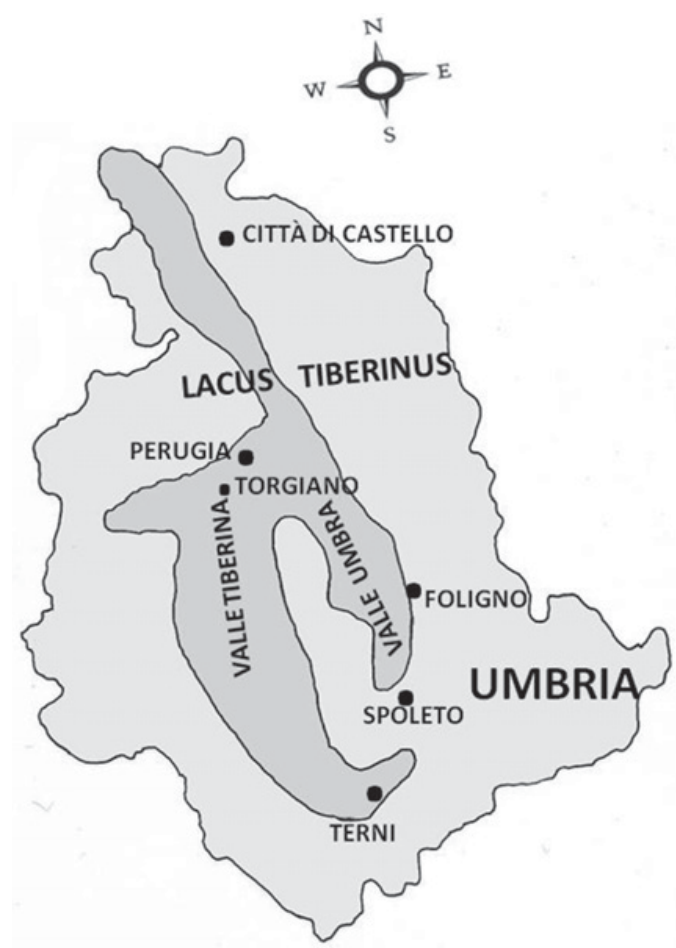

Fig. 2. Hypothetical reconstruction of the Lacus Tiberinus in the present Umbrian region.

During the Late Pliocene, due to further tectonic events and climate deterioration, the erosion accelerated and the Tiberino Lake started to dry out, until about 1.5 million years ago it turned back into a fluvial-lacustrine environment. The western branch, due to several fractures, drained quite rapidly generating the river that is now the Umbrian section of Tiber River. The eastern branch of the lake (Valle Umbra) did not have a direct effluent and drained very slowly and incompletely.

In light of recent data, this scenario appears almost unreal, and four main sectors, each with its own evolution and sedimentary sequence, are recognized: (a) North Tiber Valley, (b) Perugia-Magione and Tavernelle-Pietrafitta minor basins, (c) Valle Umbra and (d) South Tiber Valley. The eastern branch of the Tiberino basin (Valle Umbra) evolved as an endorheic, isolated basin from the Pliocene to recent times, and two main depositional cycles are recognized (Coltorti and Pieruccini, 1997; Regione Umbria, 2005), where alluvial fan deposits, lacustrine deposits and swamp deposits alternate. The lower cycle, Pliocene in age, contains the Morgnano (Spoleto) vertebrate fauna (Pantanelli, 1886; Capellini, 1888; Cappuccino et al., 2006), associated with the Triversa Faunal Unit (FU). The upper cycle was usually dated to the Early Pleistocene (Esu and Girotti, 1974, 1991; Coltorti and Pieruccini, 1997; Regione Umbria, 2005), until paleomagnetic data allowed us to extend it to the middle Pleistocene, at least in the Bevagna area (Bizzarri et al., 2011). This evolution was strongly conditioned by morphological features. The Valle Umbra, lying in a NNW-SSE direction, was surrounded by calcareous mountains to the east, by well-defined hills to the west and by a bowl-shaped rise on the south side. Waters had then (and still have) to follow the flat valley towards the north for more than $50 \mathrm{~km}$, and to overcome a threshold at Torgiano, in order to flow into the Tiber River. The threshold slowed down the flow, while the whole valley deepened because of subsidence due to fractures. In the Late Pleistocene, the lake was split into several basins of different sizes, small lakes, marshes or swamps (Colacicchi, 2010).

In Roman times there were two lakes: Lacus Umber in the plain between Assisi, Spello and Bevagna, mentioned by the poet Propertium in the Elegie (IV, 1, 24), and Lacus Clitorius that occupied the south-eastern part of the valley and was fed by Maroggia, Clitunno and Teverone Rivers (Paolo Diacono Historia Longobardorum II,16) (Fig. 3). This lake became a marsh/swamp and dried out in Republican times. During the evolution of the basin, thick layers of detritus carried by rivers and torrents from the surrounding hills, sedimented in the valley. These deposits were laid down in the plain interbedded into fine clays deposited during slow sedimentation phases. Thus, several layers of more or less coarse sand and of very permeable pebbles stratified irregularly in the plain, among the impermeable clays. The permeable layers are now outcropping at higher altitudes on the valley sides, collecting rainwater and generating deep underground aquifers blocked by the upper layers of impermeable clays. This stratigraphic situation of the Valle Umbra, combined with retarded drainage, resulted in a multitude of different water bodies, both at the surface and at groundwater level (artesian springs; L. Aiso and L. Aisillo: Colacicchi, 2010).

The Topino River basin, due to the calcareous soil and the permeable rocks of the Apennines eastern ridges, is characterized by a very high number of springs of different morphology. The Topino River also originates from several springs. Most of them feed aqueducts providing water to several Umbrian areas. The most famous are the Flaminia spring, that, due to its oligomineral bicarbonate calcium water, is bottled and is known as Nocera Umbra water, and the Angelica spring, that is known as Acqua Santa, whose therapeutic properties were known in ancient times and whose water was exported to several Italian cities as well as to Germany, Portugal and Turkey (Sigismondi, 1956).

The Topino springs are also fed by the Colfiorito karst tableland (Lippi Boncambi, 1940). Permeable rocks allow a steady and often abundant flow even to other watercourse (Menotre). The Fonti del Clitunno resurgence, where the Clitunno River rises near Campello sul Clitunno (Spoleto), are formed by underground karst water emerging through the detrital materials covering the base of the calcareous sides of the Campello mountains (Cattuto et al., 1995). The plain of Valle Umbra and the valley from Foligno to Bevagna has become greatly urbanized with widespread agriculture since ancient Roman times. In the following periods, up to 1800 , often terrible floods were observed. Therefore canalization and other anthropogenic activities have changed the 


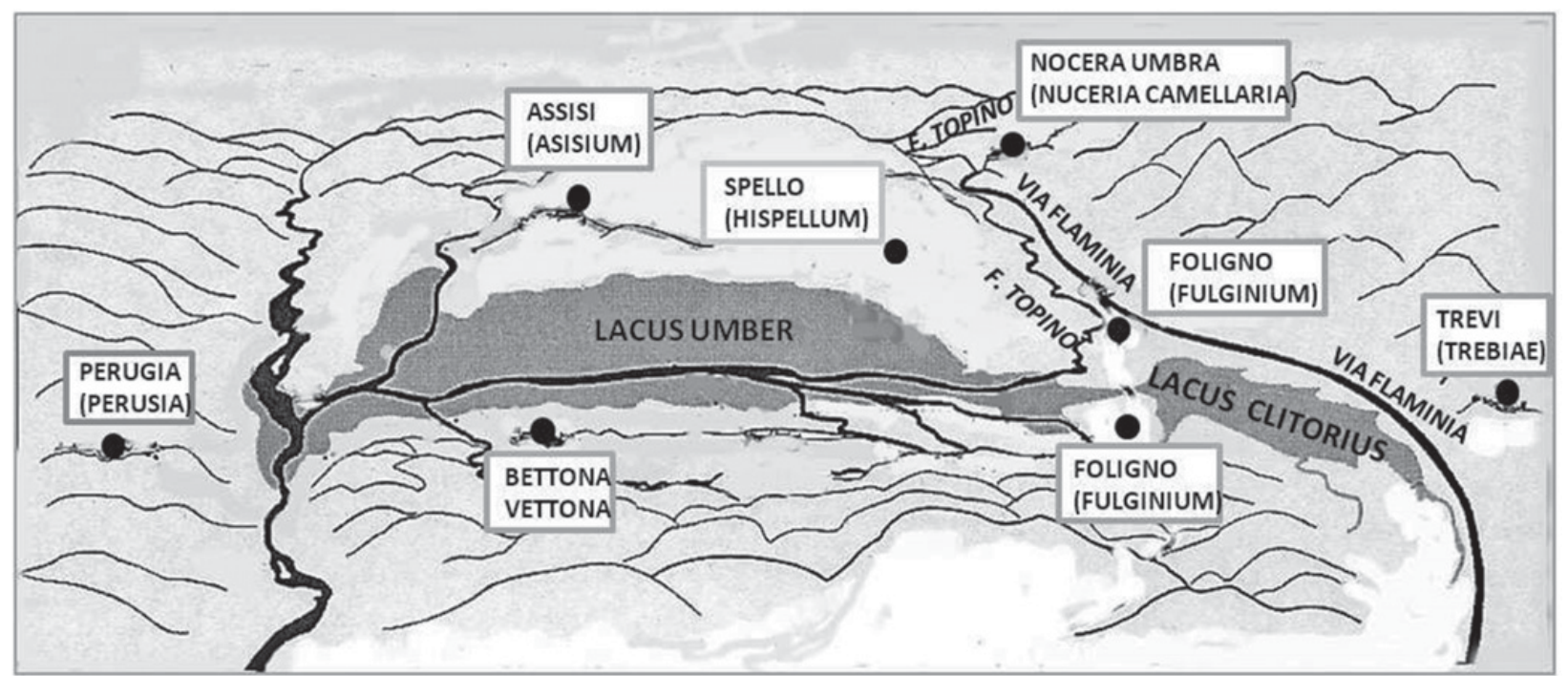

Fig. 3. Lacus Umber, Lacus Clitorius and Via Flaminia at the beginning of Roman dominion (after Pietrangeli, 1953, modified).

pristine morphology of the water courses (Colacicchi and Bizzarri, 2008). For this reason, the downstream sections (hyporhithral, potamal) of the watercourses have not been examined in this paper.

\section{Sampling sites}

The sampling sites investigated from 1947 to 2005 (Fig. 1), are listed in detail below, including additional information on catchments, locations, altitudes and longitudinal zonation of sites (Illies and Botosaneanu, 1963). Trichoptera were collected either occasionally or seasonally. The aquatic stages (larvae and pupae) were collected with hand nets or tweezers according to the habitat and typology of the substrate and were either preserved in loco in formaline $(7 \%)$ or partly reared in the laboratory until the emergence of the adults.

The adults were collected mainly during the day with an entomological net and a microaspirator in the surrounding vegetation. Only in recent times the specimens have been collected during the night with light traps (fluorescent or UV lamps). Some specimens were preserved in $70 \%$ ethanol and others were taken alive to the laboratory for dry conservation.

\section{F. Topino (48 km, 670-174 m a.s.l.)}

01: Spring La Vena, Acciano, $645 \mathrm{~m}$ a.s.1., crenal

02: Spring San Giovenale, $3 \mathrm{~km}$ upstream from Nocera Umbra, $475 \mathrm{~m}$ a.s.1., crenal

03: Spring Le Spugne, upstream from Busseto, $394 \mathrm{~m}$ a.s.1., crenal

1: Nocera Scalo, upstream from confluence with Caldognola $393 \mathrm{~m}$ a.s.1., epirhithral

2: Nocera Scalo, downstream from confluence with Caldognola $392 \mathrm{~m}$ a.s.l., detail below, including additional information on catchments, rhithral

3: Capannacce, upstream from confluence with T. Rio di Capodacqua, $392 \mathrm{~m}$ a.s.1., epirhithral
4: Poggio, downstream from confluence with T. Rio di Capodacqua, $368 \mathrm{~m}$ a.s.1., a valle abitato $374 \mathrm{~m}$ a.s.1., metarhithral

5: Valtopina, $348 \mathrm{~m}$ a.s.1., metarhithral

\section{T. Chiona (14 km, 650-197 m a.s.l.)}

1: (a) Spring del T. Chiona, downstream from Collepino, $550 \mathrm{~m}$ a.s.1., crenal

2: (b) Spring del T. Chiona, downstream from Collepino, $500 \mathrm{~m}$ a.s.l., crenal

3: (a) Downstream from Madonna di Copernieri, $489 \mathrm{~m}$ a.s.1., hypocrenal

4: $2 \mathrm{~km}$ downstream from site 1, hypocrenal

5: Fontecanale, Molinaccio, $456 \mathrm{~m}$ a.s.1., hypocrenal

6: (c) Spring del Chiona, downstream from Collepino $450 \mathrm{~m}$ a.s.l. (East Subasio), crenal

7: Tributary of T. Chiona, $410 \mathrm{~m}$ a.s.1., epirhithral

8: Downstream from Collepino, $1 \mathrm{~km}$ from site 3, 381 $\mathrm{m}$ a.s.l., epirhithral

9: $1 \mathrm{~km}$ from site 4, $377 \mathrm{~m}$ a.s.1., epirhithral

10: Passo della Paglia, upstream from confluence with

F. Topino, $13 \mathrm{~km}$ from site 1, $197 \mathrm{~m}$ a.s.1., metarhithral

\section{T. Rio Fergia $(6.8 \mathrm{~km}, 540-442 \mathrm{~m}$ a.s.l. $)$}

1: Spring $540 \mathrm{~m}$ a.s.1., crenal

2: Castello, $526 \mathrm{~m}$ a.s.l., hypocrenal

3: Boschetto, $513 \mathrm{~m}$ a.s.1., epirhithral

4: Boschetto, donwstream from trout farm, $500 \mathrm{~m}$ a.s.1., epirhithral

5: Gaifana, $480 \mathrm{~m}$ a.s.1., epirhithral

6: Upstream from Molina, $450 \mathrm{~m}$ a.s.1., epirhithral

\section{T. Caldognola (18 km, 800-392 m a.s.l.)}

1: Rivolo sorgivo, Colle Montacchiello, $710 \mathrm{~m}$ a.s.l., hypocrenal

2: Maccantone, $468 \mathrm{~m}$ a.s.l., epirhithral

3: Isola, $443 \mathrm{~m}$ a.s.1., epirhithral

4: Ponte Parrano, $424 \mathrm{~m}$ a.s.1., epirhithral

5: Nocera Scalo, $392 \mathrm{~m}$ a.s.1., epirhithral 


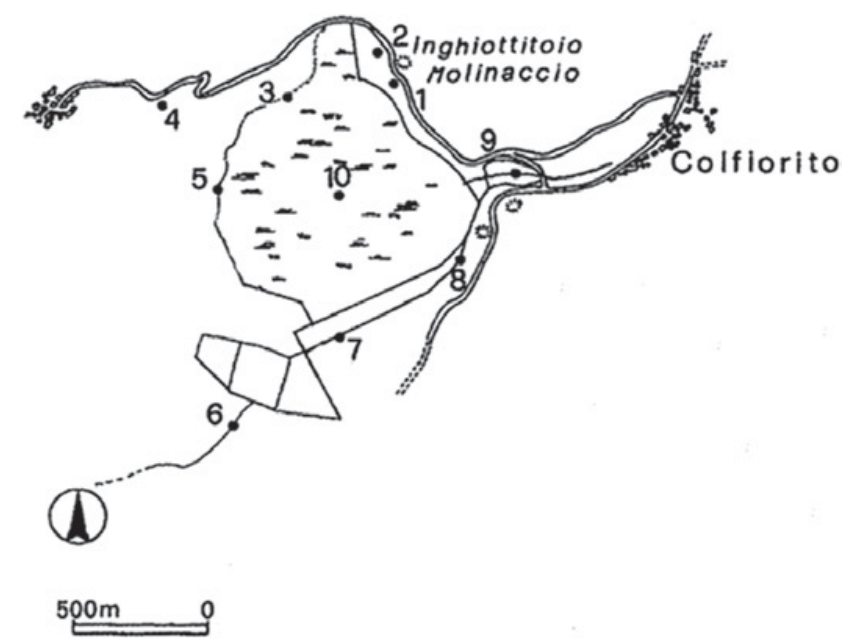

Fig. 4. Palude di Colfiorito, sampling sites: 1: Sinkhole, 2: Marsh near Sinkhole, 3: 'Chiaro', 4: 'Fontaccia', 5: Bird Observatory, 6: Ditch in SW, 7: Ex peat bog, 8: Canal under SS77 Val di Chienti, 9: Confluence of Formone Ditch, 10: Centre of marsh.

Sorgenti del Massiccio del Monte Subasio (Cianficconi et al., 2006)

1: Fonte Bregno, Assisi, $1016 \mathrm{~m}$ a.s.1., crenal

2: Spring Fosso delle Piagge: Spello, $790 \mathrm{~m}$ a.s.1., crenal

3: Fonte Orbi, Spello, $700 \mathrm{~m}$ a.s.1., crenal

4: Fonte Vecchia, Spello, $690 \mathrm{~m}$ a.s.1., crenal

5: Fosso Trosceto, Spello, $597 \mathrm{~m}$ a.s.1., hypocrenal

6: Fonte S. Giovanni, Spello, $550 \mathrm{~m}$ a.s.1., crenal

F. Menotre $(25.8 \mathrm{~km}, 850-256 \mathrm{~m}$ a.s.l)

1: San Paterniano, $850 \mathrm{~m}$ a.s.1, hypocrenal

2: Celle, $700 \mathrm{~m}$ a.s.1, epirhithral

3: Ponte S. 3: Martino, $682 \mathrm{~m}$ a.s.1., epirhithral

4: Upstream from Rasiglia, $640 \mathrm{~m}$ a.s.1., epirhithral

5: Dowstream from Rasiglia, $590 \mathrm{~m}$ a.s.1., epirhithral

6: Scopoli, $530 \mathrm{~m}$ a.s.1., epirhithral

7: Upstream from Ponte S. Lucia, $490 \mathrm{~m}$ a.s.1., epirhithral

8: Pale, $476 \mathrm{~m}$ a.s.1., epirhithral

9: Altolina, $298 \mathrm{~m}$ a.s.1., metarhithral

10: Vescia, $256 \mathrm{~m}$ a.s.1., metarhithral

Palude di Colfiorito (752 m a.s.I) (Cianficconi et al., 2000). Figure 4

1: Sinkhole

2: Marsh near Sinkhole

3: Chiaro

4: Fontaccia

5: Bird Observatory

6: Ditch in SW

7: Ex peat bog

8: Canal under SS 77 Val di Chienti

9: Confluence of Formone Ditch

10: Centre of marsh

Lago de L'Aiso (194 m a.s.l.) (Moretti, 1952; Di Giovanni et al., 1992)

1: Lago de L'Aiso, Bevagna

2: Outlet, Bevagna
Fonti del Clitunno (225 m a.s.l.) (Fig. 5)

1: Spring brooks, $225 \mathrm{~m}$ a.s.1. along the banks

2: Deep Pond, $225 \mathrm{~m}$ a.s.1. invaded by submerged hydrophytes fed by bubbling springs emerging from the bed

T. Marroggia (30 km, 600-212 m a.s.l.)

1: Spring, La Costa, $600 \mathrm{~m}$ a.s.1., crenal

2: Crocemarroggia, $580 \mathrm{~m}$ a.s.1., hypocrenal

3: Downstream from S. Giacomo $266 \mathrm{~m}$ a.s.1., epirhithral

4: $5 \mathrm{~km}$ upstream from confluence with Teverone, Trevi $212 \mathrm{~m}$ a.s.1., metarhithral (without Trichoptera)

\section{Inventory of the Trichoptera in the River Topino basin}

In the following inventory, species are ranked according to the checklist of Italian Trichoptera (Cianficconi, 2006) and to the second edition of the Atlas of European Trichoptera (Malicky, 2004).

For each species the sampling sites are recorded with the corresponding number in Figure 1, the date of the samplings in chronological order, the number of adults and aquatic stages $(1=$ larva, $\mathrm{p}=$ pupa $)$ and the name of the collector. For published records, bibliographic data are given. (L) indicates the specimens collected with light traps. Specimens identified by Moretti are kept in the G.P. Moretti collection in the Biology section of the Dipartimento di Chimica, Biologia e Biotecnologie del'Università di Perugia.

\section{Rhyacophilidae}

Rhyacophila albardana (Mc Lachlan, 1879)

Menotre: 2 - 18.II. 1971: 1 ô, Mantilacci (Di Giovanni et al., 2004); 2004-2005: 39 1, Todini; 5 - 2004-2005: 24 1, Todini.

\section{Rhyacophila dorsalis acutidens (McLachlan, 1879)}

Topino:; 01 - VI.1997: 8 1; VII.1997: 32 1; X.1997: 36 1; 03 - VI.1997: 8 1; VII.1997: 121 Cappelletti; 1 - 9.X.1994: 11 †, 5 p, 5 1; 29.XI.1994: 3 1; 3.VI.1995: 1 p, 1 1; 21.VII.1995: 5 p; 20.III.1996: 4 p, 7 1, Montanucci; 2 - 10.III.1995: 3 q; 21.V.1995: 1 p; 21.VII.1995: 1 , 6 p; 10.VIII.1995: 2 q (L), 13.X.1995: 1 ^ิ, 3 क; 20.III.1996: 4 p, 3 1, Montanucci; 3 - 8.X.1994: 1 1; 18.III.1995: 1 p, 3.VI.1995: 11 ऽึ, 1 p, 1 1; 21.VII.1995: 1 p; 25.VIII.1995:

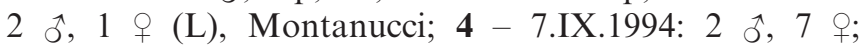
20.XI.1994: 1 ; ; 12.III.1995: 5 p, 2 1; 21.VII.1995: 1 p; 23.VIII.1995: 11 ô, 1 क, 1 p; 10.III.1996: 3 1, 4 p, Montanucci; 5 - 7.IX.1994: 4 क (L); 22.IX.1994: 1 p, 1 1; 9.X.1994: 7 p, 4 1; 12.III.1995: 3 p, 3 1; 30.IV.1995: 1 , 1 p ๙̃; 21.VII.1995: 1 p; 20.III.1996: 5 1, 1 p, Montanucci.

Chiona: 3 - 5.VIII.1996: 1 p ș; 5 - 31.VII.1995: 1 (L), Fucchi. 

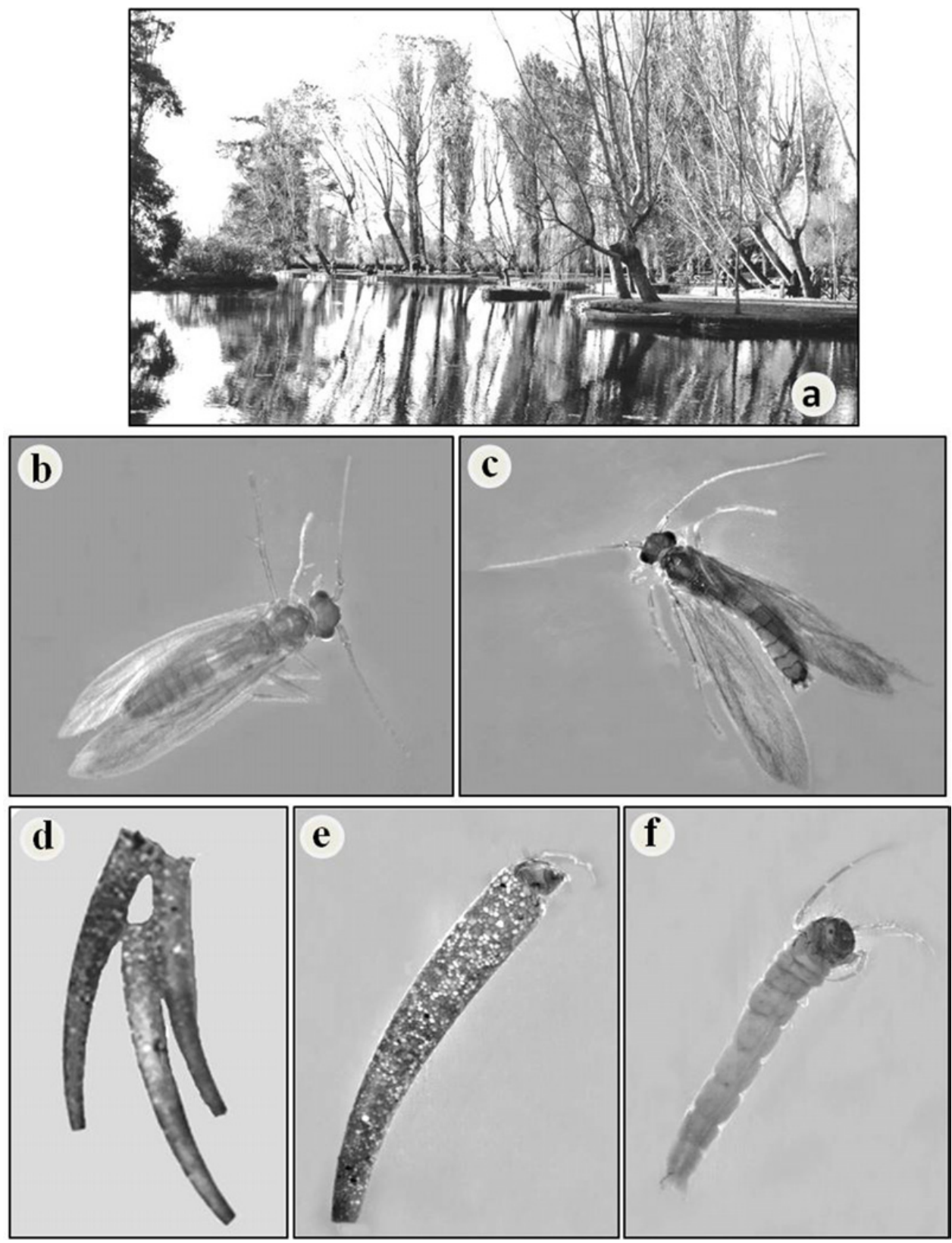

Fig. 5. Beraeodes minutus (Linnaeus, 1751) and its habitat in Fonti del Clitunno: a, Fonti del Clitunno; b and c, adult $\widehat{\delta}$, length 4-5 mm; $\mathrm{d}=3$ pupal cases; e, larva, lateral view (length $=7.5 \mathrm{~mm})$; f, larva without case, ventral view (length $=7.2 \mathrm{~mm}$ ).

Fergia: 1 - 15.X.1992: 4 ô, 2 o; 3.XI.1992: 22 ô, 2 क; 9.II.1993: 1 ô, Salerno (Di Giovanni et al., 2004); 4 - 16.IX.1997: 1 1; 5 - 16.IX.1997: 1 ô, 1 ㅇ, Farano.

Caldognola: 2 - 5.VII.1995: 1 1, Brunetti; 3 - 26.VII.1994: 6 ô, 5 \%; 11.X.1994: 1 ô; 1.IX.1995: 1 今ै. Brunetti; 4 -

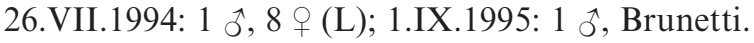

Menotre: 2 - 2004-2005: 17 1; 8 - 2004-2005: 3 1, Todini.

Marroggia: 2 - 30.V.1989: 2 1; 3 - 27, 29, 30.VII.1988: 3 ô, 1 , 4 1, Arena (Di Giovanni et al., 2004).

\section{Rhyacophila foliacea (Moretti, 1981)}

Fergia: 1 - 15.X.1992: 4 ㅅ, 2 ㅇ, 3.XI.1992: 22 ô, 2 क; 9.II.1993: 1 ô, Salerno (Di Giovanni et al., 2004); 2 - 26.X.1996: 1 p ô; 11.XII.1996: 1 p; 13.VI.1997: 1 1; 3 - 13.VI.1997: 1 p, 1 1; 6.IX.1997: 1 p; 16.IX.1997: 1 ๙, 6 p, 1 p ô, 4 1; 4-11.XII.1997: 3 p, 1 p ô; 16.IX.1997: 1 ô, 5 p ô, 4 1; 5 - 16.IX.1997: 16 ô, 2 ㅇ, 2 p oิ, 3 p ㅇ, 1 p, 2 l; 6 - 23.VII.1997: 1 ․ Farano.

Menotre: 1 - 14.X.1970: 1 ₹ै; 28.X.1970: 1 ^ै; 30.III.1971:

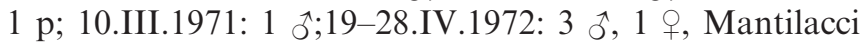
(Di Giovanni et al., 2004); 30.VII.1987: 1 3ै; 3.XI.1987:

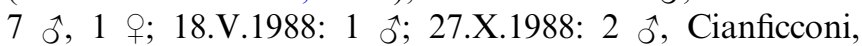


Chiappafreddo, Fortini (Di Giovanni et al., 2004); 2 - 2004 2005: 26 1, Todini; 4 - 20.X.1979: 3 个ै, 1 q, Cianficconi, Marinelli (Di Giovanni et al., 2004); 7.XII.1986: 3 1, 3 p; 3.IX.1987: 7 ô Cianficconi, Chiappafreddo, Fortini (Di Giovanni et al., 2004); 5 - 30.VII.1987: 3 ô, 1 ㅇ, 1 p; 20.X.1987: 1 o ; 3.XI.1987: 6 o; 20.VII.1988: 4 ô; 27.X.1988: $1 \hat{\jmath}, 1$, , Cianficconi, Chiappafreddo, Fortini (Di Giovanni et al., 2004); 2004-2005: 5 1, Todini; 6 - 28.X.1970: 1 ऊे; 14.XII.1970: 1 ô 10.III.1971: 1 ô, Mantilacci (Di Giovanni et al., 2004); 7 - 30.VII.1987: 3 ô, 1 क , 1 p; 3.XI.1987: 6 ô, 1 ; 26.IX.1988: 2 ๙ే; 27.X.1988: 3 ô, Cianficconi, Chiappafreddo, Fortini (Di Giovanni et al., 2004); 8 - 9.V.1967: 1 ô, Griselli (Di Giovanni et al., 2004); 2004 2005: 1 1, Todini; 10 - 27.X.1988: 2 ^ Fortini (Di Giovanni et al., 2004).

\section{Rhyacophila italica (Moretti, 1981)}

Topino: 3 - 18.III.1995: 11 วิ, Montanucci.

Chiona: 3 - 9.VIII.1995: 2 ô (L); 25.X.1995: 4 p oै; 11.VI.1996: 1 p $\widehat{\jmath}$; 5.VIII.1996: 2 p ô, Fucchi; 4 - 31.VII.1995: 3 ô, 1 + (L), Fucchi; 5 - 31.VII.1995: 1 ๙ (L); 9.VIII.1995: 20 ô (L), Fucchi; 7 - 31.VII.1995: 1 q (L), Fucchi.

Fergia: 2 - 16.III.1992: $2 \mathrm{p}$ ô, Salerno (Cianficconi et al., 1994).

Caldognola: 1 - 15.VII.1995: 1 \%; 2 - 7.V.1995: 1 \%.1 p

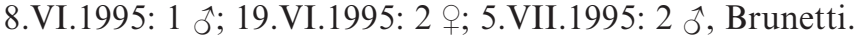

Menotre: 1 - 16.VI.1985: 8 ô, Moretti, Tucciarelli, Chiappafreddo; 28.XI.1986: 19, 1 1, Fortini (Di Giovanni et al., 2004); 3 - 18.V.1982: 1 क, Cianficconi, Chiappafreddo, Fortini (Di Giovanni et al., 2004).

Marroggia: 1 - 30.VII.1988: 1 ㅇ, Arena (Di Giovanni et al., 2004).

\section{Rhyacophila pubescens (Pictet, 1834)}

Chiona: 3 - 5.VIII.1996: 1 p $\widehat{\jmath}, 3$ 1, Fucchi.

Menotre: 1 - 14.VII.1987: 1 ô, Fortini; 30.VII.1987: 3 ô; 3.XI.1987: 1 ô, Cianficconi, Chiappafreddo, Fortini (Di Giovanni et al., 2004); 2 - 2004-2005: 4 1, Todini.

\section{Rhyacophila simulatrix (McLachlan, 1879)}

Caldognola: 5 - 22.IX.1994: 15 p; 25.IX.1994: 1 ^ิ, 1 ㅇ, Brunetti.

\section{Rhyacophila tristis (Pictet, 1834)}

Menotre: 1 - 18,19.V.1988: 2 ô, 1 ㅇ, Cianficconi, Chiappafreddo, Fortini (Di Giovanni et al., 2004); 2 - 2004-2005: 15 1; 5 - 2004-2005 1 1, Todini; 8 - 9.VI.1967; 3 ô, 1 \&, Griselli (Di Giovanni et al., 2004).

\section{Glossosomatidae}

Catagapetus nigrans (McLachlan, 1881)

Topino: 02 - 28.III.1991: 3 1, Salerno (Di Giovanni et al., 2004).

Fergia: 1 - 9.II.1991: 2 1; 24.V.1991: 1 q; 17.VII.1991: 1 人, 1 o; 16.III.1993: 1 1, Salerno (Cianficconi et al., 1994); 4.III.1997: 1 p; 9.VII.1997: 1 p; 23.VII.1997: 63 హิ, 62 +, 1 p; 2-26.X.1996: 1 1, 1 p; 4.III.1997: 1 p; 5.V.1997: 1 p; 13.VI.1997: 35 ㅇ, 18 oิ, 1 p, 1 1; 3 -9.VII.1997: 1 ડิ, 1 + , Farano.

Menotre: 1 - 30.VII.1987: 9 ô, 2 ค, 7 1; 18.V.1988: 2 ○, 1 ㅇ, 11 p, 10 1; 20.VII.1988: 2 ô, 2 o, Fortini; 27.II.1972: 1 1; 22.IX.1972: 2 p, Guidi; 2 - 2004-2005: 6 1, Todini; 5-30.VII.1987: 1 1, Fortini; 8 -9.VI.1967: 1 ô, 1 o, Griselli (Di Giovanni et al., 2004).

\section{Agapetus nimbulus (McLachlan, 1879)}

Fonte Orbi: 16.IV.1992: 1 1, Cardinali.

Menotre: 2 - 2004-2005: 9 1, Todini; 4 - 11.V.1987: 4 1, 553 p; 30.VII.1987: 3 oิ, 2 क , Fortini; 5 - 12.III.1987: 1 p, Fortini.

Clitunno: 1 - 24.III.1948: 198 p, 78 1; 5.V.1948: 1 ^ Moretti (Moretti, 1952); 31.III.1952 - 20.IX.1952: 4 ㅇ, 2879 p, Casagrande (Moretti, 1952); 24.VII.1953: 1 ô, 1 \%, Bellini (Di Giovanni et al., 2004); 23 IV 19676 p, 1 1, Griselli (Di Giovanni et al., 2004); 18.IX.1972: 3 수 15.X.1972: 7 구, 10 ○; 19.XI.1972: 1 ค; 23, 25.III.1973: 1 ô,1 1 ; 5.V.1973: 16 ô, 16 o; 30.V.1973: 2 o; 30.VII.1973: 4 o, 4 o , Corvelli.

\section{Hydroptilidae}

Stactobia caspersi (UImer, 1950)

Sorgente Fosso delle Piagge: 12.V.1992: 1 p; 11.IV.1993: 11 , Cardinali.

\section{Oxiethira flavicornis (Pictet, 1834)}

Lago de L'Aiso: 2 - XI.1991: 1 1, Di Giovanni (Di Giovanni et al. 1992).

\section{Hydroptila angulata (Mosely, 1922)}

Topino: 2 - 10.VIII.1995: 2 ㅇ (L), Montanucci; 4 - 23.VIII.1995: 3 †; 5 - 7.IX.1994: 24 ภิ, 19 ๆ (L), Montanucci.

Palude di Colfiorito: 2 - 3.VIII.1994: 6 (L); 5 - 6.IX.1998: 2 ㅇ (L), Cianficconi, Mazzerioli, Salerno (Cianficconi et al., 2000).

Lago de L'Aiso: 1 - 1952: 4 ऽૈ, 6 ㅇ, Moretti (Di Giovanni et al., 2004). 
Hydroptila forcipata (Eaton, 1873)

Topino: 5 - 7.IX.1994: 16 ㄱ, 68 (L), Montanucci.

Palude di Colfiorito: 2 - 7.IX.1993: 1 ô( (L), Cianficconi, Mazzerioli, Salerno (Cianficconi et al., 2000).

\section{Hydroptila insubrica (Ris, 1903)}

Chiona: 4 - 9.VIII.1995: $3 \hat{\jmath}$ (L), Fucchi.

Menotre: 5 - 30.VII.1987: 5 ô, 1 q, Cianficconi, Chiappafreddo, Fortini (Di Giovanni et al., 2004); 9 - 30.VII.1987: 5 ô, 2 †, Cianficconi, Chiappafreddo, Fortini (Di Giovanni et al., 2004).

\section{Hydroptila martini (Marshall, 1977)}

Clitunno: 1 - 3.VI.1952: 3 ô, 41 क; 5.VIII.1952: 7 कै;

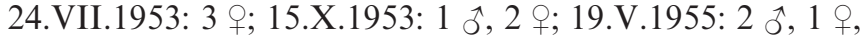
Bellini (Di Giovanni et al., 2004); 20.IX.1952: 35 , Casagrande (Di Giovanni et al., 2004); 5, 6.VIII.1952: 13 ô, 3 ㅇ, 31 p, 11 1; 23.V.1953: 148 ô, 106 우 23.VII.1953: 109 क; 15.X.1953: 1 ô, 2 क, Moretti (Di Giovanni et al., 2004); 30.IV.1973: 4 +, Corvelli (Di Giovanni et al., 2004); 22.X.1973: 2 o, 4 क , Cianficconi (Di Giovanni et al., 2004); 1.VI.1979: 6 ô 1 o, Moretti, Cianficconi, Tucciarelli, Marini (Di Giovanni et al., 2004).

\section{Hydroptila occulta (Eaton, 1873)}

Topino: 2 - 10.VIII.1995: 2 ㅇ (L), Montanucci; 4 - 23.VIII.1995 2 + , Montanucci.

Chiona: 3 - 9.VIII.1995: 1 ô (L), Fucchi.

Clitunno: 1 - 3.VI.1952: 3 ๙ै, 40 क; 5.VIII.1952: 12 oै, 8 q, Moretti (1952).

Hydroptila ruffoi (Moretti, 1981)

Fonte Orbi: 19.V.1990: 6 ๙ิ, 2 ค, Romozzi.

Hydroptila sparsa (Curtis, 1834)

Lago de L'Aiso: 1 - 1952: 1 హิ, Moretti (1952).

Hydroptila tineoides (Dalman, 1819)

Menotre: 2 - 2004-2005: 9 1, Todini.

\section{Hydroptila uncinata (Morton, 1893)}

Menotre: 5 - 2004-2005: 10 1; 8 - 2004-2005: 6 1, Todini; 9 -30.VII.1987: 1 今, Cianficconi, Chiappafreddo, Fortini (Di Giovanni et al., 2004).

\section{Allotrichia pallicornis (Eaton, 1873)}

Menotre: 7 - 30.VII.1987: 1 ô, Cianficconi, Chiappafreddo, Fortini (Di Giovanni et al., 2004); 9 - 30.VII.1987: 1 ô, 2 + Cianficconi, Chiappafreddo, Fortini (Di Giovanni et al., 2004).

\section{Philopotamidae}

Wormaldia mediana (McLachlan, 1878)

Chiona: 3 - 9.VIII.1995: 1 ô, (L) Fucchi; 4 - 31.VII.1995: 1 oै (L), Fucchi.

Fergia: 2-13.VIII.1992: 1 ô, Salerno (Cianficconi et al., 1994).

Menotre: 5 - 2004-2005: 2 1, Todini.

\section{Wormaldia occipitalis (Pictet, 1834)}

Chiona: 3 - 9.VIII.1995: 1 ^ (L), Fucchi; 5 - 9.VIII.1995: 1 ภै, 1 क (L); 9.X.1995: 2 ㅇ (L), Fucchi.

Menotre: 0 - 30.VII.1987: 1 q, Fortini; 1 - 20.X.1987: 1 ㄱ;

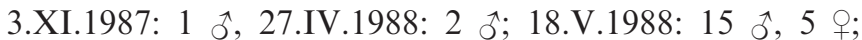

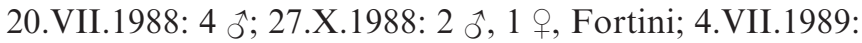
1 ô, Cianficconi, Chiappafreddo (Di Giovanni et al., 2004).

Marroggia: 1 - 10.XII.1986: 2 ㅇ, 2 1; 11.II.1987: 1 कै; 21.VIII.1987: 1 ๙ै; 24.XI.1987: 1 क, 1 1; 1.X.1989: 1 ô, 1 \%, 2 1, Arena (Di Giovanni et al., 2004); 3 - 9.III.1987: 1 , 11 Arena (Di Giovanni et al., 2004).

Wormaldia pulla marlieri (Moretti, 1981)

Fergia: 3 - 24.V.1991: 1 ô, Salerno (Cianficconi et al., 1994).

\section{Hydropsychidae}

\section{Hydropsyche instabilis (Curtis, 1834)}

Topino: 02 - 2.VII.1979: 2 + , Lipparelli.

Caldognola: 3 - 7.V.1995. 2 1; 15.VII.1995: 1 ศే; 5 26.VII.1994: 4 ㅇ; 7 - 26.VII.1994: 108 ऽิ, 453 우 (L), Brunetti.

Fergia: 2-17.VII.1991: 1 ô, Salerno (Cianficconi et al., 1994).

Menotre: 2 - 2004-2005: 8 1; 5 - 2004-2005: 35 1, Todini.

\section{Hydropsyche klefbecki (Tjeder, 1946)}

Topino: 2 - 21.VII.1995: 11 ภ; 10.VIII.1995: 2 ○ึ(L), Montanucci. 
Menotre: 1 - 28.XI.1986. 1 1, Fortini; 2 - 2004-2005. 21 Todini; 5 - 30.VII.1987: 2 ô, 2 ค, 1 p ô, Cianficconi, Chiappafreddo, Fortini (Di Giovanni et al., 2004); 20042005. 11 1, Todini; 7 - 30.VII.1987: 2 ô, 2 ㅇ, 1 p , Cianficconi, Chiappafreddo, Fortini (Di Giovanni et al., 2004).

\section{Hydropsyche modesta (Navas, 1925)}

Menotre: 5 - 2004-2005: 12 1, Todini.

\section{Hydropsyche pellucidula (Curtis, 1834)}

Topino: 2 - 20.III.1996: 3 1, Montanucci; 3 - 21.VII.1995: 1 ô; 20.III.1996: 2 1, Montanucci; 4 - 23.VIII.1995: 9 q, 20.III.1996: 2 1, Montanucci; 5 - 1.IX.1995: 7 oิ, 9 q (L); 20.III.1996: 2 1, Montanucci.

Menotre: 1 - 18.V. 1988: 1 §ै, Cianficconi, Chiappafreddo, Fortini (Di Giovanni et al., 2004); 16.VI.1985: 9 , Moretti; 2 - 2004-2005: 3 1, Todini; 3 - 27.X.1971: 1 1; 27.X.1971: 1 1; 27.XII.1971: 11 1; 27.IV.1972: 1 1; 28.VI.1972: 1 1; 27.VII.1972: 1 1; Guidi; 4 - 7.XII.1986: 1 1, Fortini; 27.X.1971: 1 1; 27.XI.1971: 1 1, Guidi; 5-2004: 48 1, Todini; 8-2004-2005: 28 1, Todini; 9-11.V.1987: 1 1; 14.VII.1987: 1 1, Fortini; 10 - 27.XII.1971: 1 1; 27.III.1972: 1 p; 27.IV.1972: 1 1; 28.V.1972: 1 1; 27.VII.1972: 1 1; 27.VIII.1972: 1 1; 22.IX.1972: 1 1, Guidi.

Marroggia: 2 - 30.V.1989: 1 Arena (Di Giovanni et al., 2004); 3 - 12.IV.1987: 2 ๙ิ, 1 1; 26.IV.1988: 1 ऽิ, 1 ㅇ, 2 1, Arena (Di Giovanni et al., 2004).

\section{Hydropsyche spiritoi (Moretti, 1991)}

Chiona: 9 - 31.VII.1995: 6 ô, 10 q (L), Fucchi.

Fergia: 2 - 22.VI.1991: 1今ึ, Salerno (Cianficconi et al., 1994).

$$
\text { Hydropsyche tenuis (Navas, 1932). }
$$

Fergia: 3 - 19.VIII.1997: 1ðิ, 3ำ (L), Salerno.

\section{Cheumatopsyche lepida (Wallengren, 1891)}

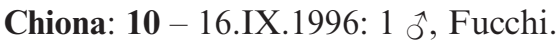

Marroggia: 2 - 2.IV.1989: 1 ㅇ, 1 1, Arena (Di Giovanni et al., 2004).

\section{Polycentropodidae}

\section{Plectrocnemia conspersa (Curtis, 1834)}

Chiona: 3 - 9.VIII.1995: 2 oิ (L); 8.IX.1995. 1 के (L); 16.IX.1996: 1 คิ, 1 p ㅇ, Fucchi; 5 - 9.VIII.1995: 1 ô (L), Fucchi.
Rio Fergia:- 3: 13.IX.1992: 1 +, Salerno (Cianficconi et al., 1994).

Menotre: 3 - 2004-2005. 3 1, Todini.

Marroggia: 1 - 21.I.1990: 1 ô, 3 1, Arena (Di Giovanni et al., 2004).

\section{Plectrocnemia geniculata corsicana (Mosely, 1930)}

Topino: 01 - X.1997: 2 1, Cappelletti.

Chiona: 3 - 10.X.1995: 2 ô (L); 5.VIII.1996: 2 1, Fucchi.

Fonte Bregno: 10.VI.1992: 1 ภ̊; 24.IV.1993: 1 1, Cardinali.

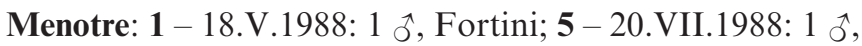
Fortini.

\section{Polycentropus mortoni (Mosely, 1930)}

Topino: 5 - 7.IX.1994: 1 ऽิ, 5 + , Montanucci.

Menotre: 4 - 7.XII.1986: 1 p, Fortini; 9-30.VII.1987: 1 今, Fortini.

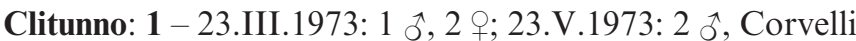
(Di Giovanni et al., 2004); 11.X.1953: 1 ô, Casagrande; 11.X.1953: 1 ^ै; 15.X.1953: 1 क्; 23.V.1953: 2 o, Bellini (Di Giovanni et al., 2004); 18.IX.1972: 1 कै; 15.X.1972: 1 कै,

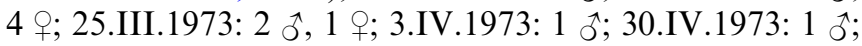

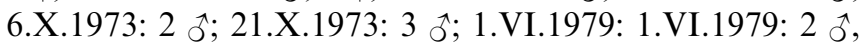
Moretti, Cianficconi, Marini (Di Giovanni et al., 2004).

Marroggia: 1 - 8.III.1987: 1 ô; 3 - 9.III.1987: 1 ㅇ, 1 1, Arena (Di Giovanni et al., 2004).

\section{Psychomyidae}

\section{Psychomyia pusilla}

Lago dell'Aiso: 2 - 6.VII.1952: 1 adult, Moretti (1952).

\section{Lype reducta}

Fergia: 2 - 22.VII.1991: 1 ô, Salerno (Cianficconi et al., 1994).

Fonte Orbi: 19.V.1990: 2 ๙ै, Romozzi.

Tinodes antonioi (Botosaneanu and Vigano, 1974)

Topino: 5 - 7.IX.1994: 1 (L), Montanucci.

Chiona: 3 - 9.VIII.1995: 43 ㅇ (L); 16.IX.1996: 1 ô, 1 , Fucchi; 5 - 31.VII.1995: 1 ô, 1 क (L); 9.VIII.1995: 1 ô, 3 ㅇ (L), Fucchi.

Fergia: 1 - 23.VII.1997: 1 ô, Farano. 
Sorgente Fonte Vecchia: 28.IV.1992: 1 1; 05.V.1993: 1 ô, Cardinali.

Sorgente del Fosso Trosceto: 12.V.1992: 1 ô, 1 1; 11.IV.1993: 1 1, Cardinali.

Fonte S. Giovanni: 16.IV.1992: 1 1; 8.V.1993: 1 ô, 1 1, Cardinali.

Menotre: 1 - 30.VII.1987: 1 , Cianficconi, Chiappafreddo, Fortini (Di Giovanni et al., 2004); 2 - 2004-2005: 13 1, Todini; 5: 24.VII.1978: 1 ô, Moretti, Cianficconi, Marinelli (Di Giovanni et al., 2004); 8 - 2004-2005: 7 1, Todini; 9 - 30.VII.1987: 1 q, Cianficconi, Chiappafreddo, Fortini (Di Giovanni et al., 2004).

\section{Tinodes maclachlani (Kimmins, 1966)}

Chiona: 3 - 11.VI.1996: 1 1; 5.VIII.1996: 4 1, Fucchi; 8 - 5.VIII.1996: 1 1, Fucchi.

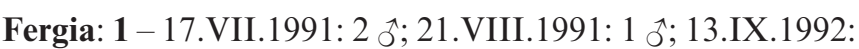

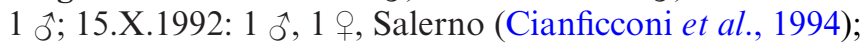
2-26.X.1996: 1 1, Salerno.

Menotre: 2 - 2004-2005: 5 1, Todini.

Clitunno: 1 - 21.IV.1967: 4 ŝ,Gammaitoni, (Di Giovanni et al., 2004); 1.VI.1979: 1 ô Moretti, Cianficconi, Marini (Di Giovanni et al., 2004); 23.V.1953: 1 ô, Casagrande (Di Giovanni et al., 2004); 15.X.1972: 1 ô, 1 ค, Moretti;

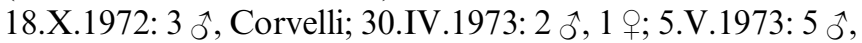
1 o; 30.V.1973: 2 ô, 1 क; 21.X.1973: 2 ô, 3 क, Moretti; 1.VI.1979: 1 ô, Cianficconi.

\section{Tinodes waeneri (Linnaeus, 1758)}

Menotre: 5 - 2004-2005: 1 1, Todini.

\section{Ecnomidae}

Ecnomus tenellus (Rambur, 1842)

Menotre: 5 - 17.VII.1991: 1 ๙ै, 1 \&, Salerno.

\section{Phryganeidae}

\section{Agrypnia varia (Fabricius, 1793)}

Palude di Colfiorito: 1 - 25.III.1996: 1 1; 6.V.1996: 1 1; 19.IV.1997: 1 1; 6.IX.1998: 1우 (L), Mazzerioli, Salerno (Cianficconi et al., 2000); 2-7.IX.1993: 1 ô, 6 + (L), Salerno, Tavone; 29.VI.1994: 2 ㅇ (L), Cianficconi, Mazzerioli, Moretti, Salerno, Speziale; 3.VIII.1994: 1 ô (L), Cianficconi, Mazzerioli, Moretti, Salerno; 19.VI.1996: 1 우 (L); 6.VIII.1996: 1 ㅇ (L) Mazzerioli, Salerno (Cianficconi et al., 2000); 3-27.V.1998: 1 ô, 1 p, Cianficconi, Corallini, Salerno, Tucciarelli (Cianficconi et al., 2000); 5-19.VI.1996: 1 ô, 1 ㅇ (L); 6.VIII.1996: 2 ㅇ (L); 10.VI.1998: 1 ㅇ (L), Mazzerioli, Salerno (Cianficconi et al., 2000).

\section{Brachycentridae}

Micrasema dolcinii (Botosaneanu and Moretti, 1986)

Menotre: 1 - 28.XI.1986: 1 p, Fortini; 30.VII.1987: $12 \hat{\jmath}, 3$ \&, Cianficconi, Chiappafreddo, Fortini; 11.V.1987: 153 1, Fortini (Di Giovanni et al., 2004); 2 2004-2005: 21 1, Todini; 4 - 16.VI.1987: 1 ऊै, 3 o f Fortini (Di Giovanni et al., 2004); 5 - 24.VII.1978: 13 s, 7 ㅇ, Moretti and coll.; 16.VI.1987: 3 ô, 2 क; 20.VII.1988: 25 ô, 14 ․, Fortini (Di Giovanni et al., 2004); 2004-2005: 12 1, Todini; 7 - 14.VII.1987: 2 ô, Fortini (Di Giovanni et al., 2004).

\section{Micrasema minimum (McLachlan, 1876)}

Menotre: 5 - 24.VII.1978: 4 今ै, Moretti (Di Giovanni et al., 2004); 2004-2005: 1 1, Todini; 8 - 09.VI.1967: 1 ô, 6 o , Griselli (Di Giovanni et al., 2004).

\section{Micrasema morosum (McLachlan, 1868)}

Menotre: 2 - 2004-2005: 19 1, Todini.

\section{Limnephilidae}

\section{Drusus camerinus (Moretti, 1981)}

Menotre: 0 - 30.VII.1987:1 1, Fortini; 04.VII.1989: 1 సै, 1 1, Moretti, Cianficconi, Chiappafreddo, Speziali (Di Giovanni et al., 2004); 1 - 3.XI.1987: 1 ^ै; 27.IV.1988: 7 ô, 1 o; 18.V.1988: 4 วิ, 1 क; 20.VII.1988: 2 क, Cianficconi, Chiappafreddo, Fortini (Di Giovanni et al., 2004); 5 - 4.VII.1989: 3 1, Moretti, Cianficconi, Chiappafreddo (Di Giovanni et al., 2004); 7 - 29.V.1972: 1 ô, 9 1, Guidi; 8 -9.VI.1967: 1 oै, Griselli (Di Giovanni et al., 2004).

\section{Drusus improvisus (McLachlan, 1884)}

Topino: 02 - 11.II.1951: 2 ô, Moretti; 9.XI.1952: 1 ㅇ, Gianformaggio (Di Giovanni et al., 2004); 6.I.1980: 6 1, Lipparelli.

\section{Limnephilus auricula (Curtis, 1834)}

Palude di Colfiorito: 2 - 20.V.1951: 1 ô; 21.VI.1951:

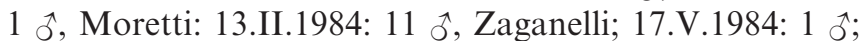
Pirisinu, 19.VI.1996: 1 + , Mazzerioli, Salerno (Cianficconi et al., 2000).

\section{Limnephilus bipunctatus (Curtis, 1834)}

Palude di Colfiorito: 1 - 19.VI.1996: 1 q, Mazzerioli, Salerno (Cianficconi et al., 2000); 2 - 7.V.1967: 1 ô, 2 , Cianficconi, Pirisinu, V.1979: 1 p, 1 1, Principato; 5.IV.1982: 2 ^ิ, 1 1, Zaganelli (Cianficconi et al., 2000); 
5 - 25.III.1996: 3 o, 1 1; 17.V.1998: 1 ๙ิ, 1 p Mazzerioli, Salerno (Cianficconi et al., 2000); 7 - 20.V.1951: 4 ô, 1 우, Moretti (Cianficconi et al., 2000).

\section{Limnephilus flavicornis (Fabricius, 1787)}

Palude di Colfiorito: 1 - 6.V.1967: 1 1; 09.V.1967: 2 ऽิ, 1 ㅇ, Cianficconi; 27.IV.1983: 5 ș, 1 ㅇ, Mearelli; 6.V.1996: 1 p;

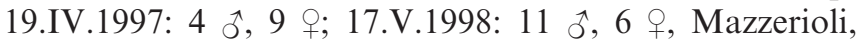
Salerno; 27.V.1998: 1 ô, 1 p, Mazzerioli, Salerno (Cianficconi et al., 2000); 14.III.1999: 1 1, Cianficconi, Mazzerioli, Salerno (Cianficconi et al., 2000); 2 - V.1979: 1 1, Principato; 6.V.1996: 6 \&, 1 1, Mazzerioli, Salerno; 3 - 27.V.1998: 12 ș, 9 ㅇ, Mazzerioli, Salerno (Cianficconi et al., 2000); 5 - 25.III.1996 1 ô, 1 1; 17.V.1998: 3 ô, 6 q Mazzerioli, Salerno (Cianficconi et al., 2000); 7 - 21.VI.1951: 4 ô, 16 o (L), 10 ô, 10 ㅇ, Moretti; 14.III.1999: 1 1, Corallini, Mazzerioli, Salerno (Cianficconi et al., 2000); 9-9.IV.1997: 1 ㅇ, 1 p, 1 1, 9.XI.1998: 1 ô, 2 ㅇ, Mazzerioli, Salerno (Cianficconi et al., 2000).

\section{Limnephilus helveticus (Schmid, 1965)}

Marroggia: 3 - 10.V.1987: 1 o, 1 1, Arena (Di Giovanni et al., 2004).

\section{Limnephilus hirsutus (Pictet, 1834)}

Palude di Colfiorito: 4 - 17.V.1998: 4 ๙ึ, 1 ๆ, 1 p, Mazzerioli, Salerno; 14.III.1999:1 1, Cianficconi, Mazzerioli, Salerno (Cianficconi et al., 2000).

\section{Limnephilus ignavus (McLachlan, 1865)}

Palude di Colfiorito: 4 - 17.V.1998: 2 q, 1 p, 1 1, Mazzerioli, Salerno (Cianficconi et al., 2000); 9 - 9.XI.1998: 1 , Mazzerioli, Salerno; 14.III.1999: 1 1, Cianficconi, Mazzerioli, Salerno (Cianficconi et al., 2000).

\section{Limnephilus Iunatus (Curtis, 1834)}

Chiona: 9-9.V.1995: 1 , Fucchi; 10-25.X.1995: 1 ๙ิ, 1 ㅇ (L), Fucchi.

Menotre: 4 - 16.VI.1987: 1 ڤै, Fortini (Di Giovanni et al., 2004).

Palude di Colfiorito: 1 - 25.III.1996: 1 ô, 1 q, 1 1; 17.V.1998: 2 ô, 3 o; 10.VI.1998: 1 +, Mazzerioli, Salerno (Cianficconi et al., 2000); 2 - 25.III.1996: 1 1; 6.V.1996: 1 ㅇ, 1 p, Mazzerioli, Salerno (Cianficconi et al., 2000); 5 - 25.III.1996: 1 1, Mazzerioli, Salerno (Cianficconi et al., 2000); 6 - 19.IV.1997: 1 q, 1 1, Mazzerioli, Salerno;

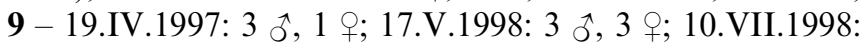
4 ô, 1 o; 9.XI.1998: 4 q, Mazzerioli, Salerno; 14.III.1999: 1 1, Cianficconi, Mazzerioli, Salerno (Cianficconi et al., 2000).

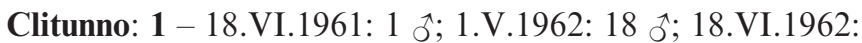
4 q, Moretti, 10.V.1969: 1 q, Corvelli; 5.VI.1969: 1 q, Pirisinu (Di Giovanni et al., 2004).

Marroggia: 1 - 2.II.1987: 1 ô, 1 1; 8.III.1987: 1 ๙ิ, 1 1; 27.III.1988: 2 o; 2.IV.1989: 1 ふै, 1 1, Arena (Di Giovanni et al., 2004); 2 -21.I.1990: 1 q, 1 1; 29.IV.1990: 1 ふै, 4 q, 4 1, (Di Giovanni et al., 2004); 3 - 12.IV.1987: 6 ^ิ, 8 क , 6 1;

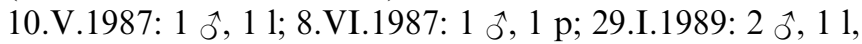

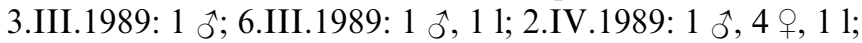
29.IV.1990: 6 + , 3 1; 4.VI.1990: 1 , 1 1, Arena (Di Giovanni et al., 2004).

\section{Limnephilus rhombicus reseri (Malicky, 1985)}

Palude di Colfiorito: 1 - 25.III.1996: 3 sै; 6.V.1996: 1 1; 19.IV.1997: 1 p, 1 1; 17.V.1998: 1 p, Mazzerioli, Salerno (Cianficconi et al., 2000); 2 - V.1979: 1 1, Principato; 25.III.1996: 1 1; 6.V.1996: 2 ô, 1 p, 1 1, Mazzerioli, Salerno (Cianficconi et al., 2000); 5 - 6.V.1996 1 p 1, 1 p, Mazzerioli, Salerno; 8 - 25.III.1996 1 1, Mazzerioli, Salerno (Cianficconi et al., 2000); 9 - 19.IV.1997 1 1, Mazzerioli Salerno (Cianficconi et al., 2000).

Clitunno: 1 - 8.V.1948: 6 ふै, 10 o; 2 1, 23.V.1953: 1 क; 1.VI.1962: 1 +, Moretti (Di Giovanni et al., 2004); 19.IV.1955: 2 ô, 1 o; 20.V.1958: 1 ㅅ, 1 o, Bellini (Di Giovanni et al., 2004); 9-26.V.1964 9 ภ, 19 ㅇ, Pucci (Di Giovanni et al., 2004); 25.V.1964: 5 ô; 9.V.1970: 105 1, Moretti, Cianficconi (Di Giovanni et al., 2004); 14 - 31.III.1966: 6 ઈิ, 2 o, 1 p; 12.IV.1970: 1 శ,

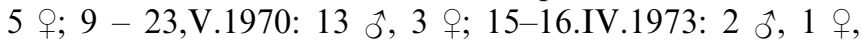
Cianficconi (Di Giovanni et al., 2004); 27.IX.1972: 1 , Corallini (Di Giovanni et al., 2004); 27.V.1981: 3 ŝ, 9 +; 14. VI. 1981: 2 1, Chiappafreddo (Di Giovanni et al., 2004); 15.IV.1971: 1 ô, Sbrenna (Di Giovanni et al., 2004).

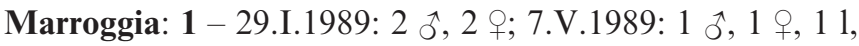
(Di Giovanni et al., 2004); 2 - 29.I.1989: 4 ô, 7 q, 6 1; 21.I.1990: 1 ô, 1 1; 27.I.1991: 1 ô, 1 1, (Di Giovanni et al., 2004); 3-10.V.1987: 1 1; 2.IV.1989: 1 ô, 1 1; 21.I.1990: 1 , 1 1, Arena (Di Giovanni et al., 2004).

\section{Glyphotaelius pellucidus (Stephens, 1833)}

Topino: 03 - VII.1997: 4 1, Cappelletti

Palude di Colfiorito: 1 - 25.III.1996: 3 ๙̃; 6.V.1996: 1 1, 19.IV.1997: 1 p, 1 1; 17.V.1998: 1 p, Mazzerioli, Salerno (Cianficconi et al., 2000).

\section{Grammotauliuus nigropunctatus (Retzius, 1783)}

Topino: 01 - I.1998: 4 1, Cappelletti

Palude di Colfiorito: 1 - 25.III.1996: 1 1; 6.V.1996: 1 1; 19.IV.1997: 1 1, Mazzerioli, Salerno (Cianficconi et al., 2000); 2 - 16.IV.1967: 2 क, Gianotti; 7.V.1967: 5 ô, Cianficconi, Pirisinu; 5 - 25.III.1996: 1 1; 6.V.1996: 1 1, Mazzerioli, Salerno (Cianficconi et al., 2000); 
10 - 10.III.1951: 1 1, Moretti, Secondari (Cianficconi et al., 2000).

\section{Potamophylax cingulatus gambaricus (Malicky, 1971)}

Topino: 01-5.X.1979: 1 ô, Lipparelli (Di Giovanni et al., 2004); 1 - 9.X.1994: 11 ơ; 18.III.1995: 11 ㅇ, Montanucci; 2 - 18.III.1995: 1 o, Montanucci; 3 - 8.X.1994: 11 \%; 29.XI.1994: 11 ô, Montanucci; 4 - 20.XI.1994: 11 సै; 12.III.1995: 2 क; 21.V.1995: 1 ô, 1 ㅇ, Montanucci; 5 - 22.IX.1994: 6 p, 1 1; 9.X.1994: 2 ô, 1 ㅇ, 5 p, 6 1; 21.VII.1995: 22 +, Montanucci.

Chiona: 1 - 17.III.1992: 1 ô, 1 q, 1 1; 30.III.1993: 11 , Cardinali; 2 - 17.III.1992: 1 1, Cardinali; 3 - 9.VIII.1995: 1 ô (L); 10.X.1995: 3 오 (L); 25.X.1995: 4 p ô; 11.VI.1996: 5 1; 5.VIII.1996: 5 1; 16.IX.1996: 1 p ô, 1 p + , Fucchi;

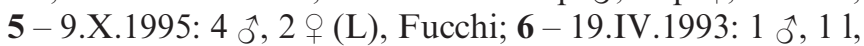
Cardinali.

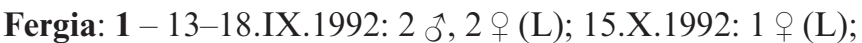
3.XI.1992: 6 ㅇ (L); 16.III.1993: 5 1, Salerno (Cianficconi et al., 1994); 21.I.1997: 1 1; 9.VII.1997: 1 p, 1 1; 3 - 13.VI.1997: 2 1; 9.VII.1997: 1 1; 6.IX.1997: 1 p;

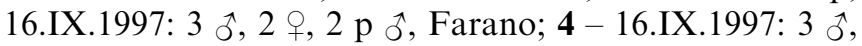
1 +, $1 \mathrm{p}$ ô, $1 \mathrm{p}$, Farano.

Caldognola: 1 - 7.V.1995: 1 1; 2-5.VII.1995: $1 \mathrm{p}$ ㅇ, 3 p $\widehat{\jmath}$, 5 3ै, 8 क; 10.X.1995: 3 क; 12.XII.1995: 1 1; 3 - 5.VII. 1995: 1 1; 25.VII.1995: 1 1; 1.IX.1995: 1 ô, 1 o , 11 1, Brunetti;

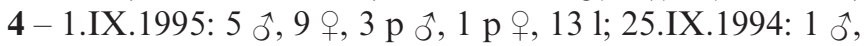
3 ㅇ, 1 1; 19.III.1995: 1 1; 7.V.1995: 7 1; 18.VII.1995: 24 1; 1.IX.1995: 7 ^ै, 1 ㅇ, 1 p ; 12.X.1995: 1 ㅇ, Brunetti.

Fonte Orbi: 16.IV.1992: 1 ô, 2 \&, 1 1, Cardinali.

Sorgente del Fosso Trosceto: 12.V.1992: 2 ค, 1 1; 8.VII.1992: 1 1; 24.X.1992: 1 1, Cardinali.

Menotre:1 - 11.V.1987: 11, Fortini; 2 - 2004-2005: 25 1, Todini; 4 - 11.V.1987: 40 1; 14.VII.1987: 36 1; 3.IX.1987: 1 sิ, Fortini (Di Giovanni et al., 2004); 7 - 11.V.1987: 1 1; 26.IX.1988: 1 今ึ, Fortini; 8-2004-2005: 50 1, Todini.

Halesus appenninus (Moretti and Spinelli, 1981)

Topino: 01 - VII.1997: 2 1, Cappelletti; 1 - 9.X.1994: 1 p $\circ$; 3.VI.1995: 6 1; 21.VII.1995: 3 1, Montanucci;

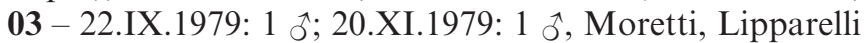
(Di Giovanni et al., 2004); IV.1997: 12 1; I.1998: 41 Cappelletti,; 2 - 21.VII.1995: 11 ơ; 20.III.1996: 31 , Montanucci; 3 - 8.X.1994: 12 ô, 12 o, 2 1; 21.VII.1995: 2 1; 20.III.1996: 3 1, Montanucci; 4 - 12.III.1995: 11 के; 20.III.1996: 3 1, Montanucci; 5 - 9.X.1994: 2 p क; 12.III. 1995: 1 1; 30.IV.1995: 1 1; 21.VII.1995: 1 §ै, Montanucci.

Chiona: 3 - 5.VIII.1996: 1 1, Fucchi.

Fergia: 2 - 9.VII.1997: 11 Farano,; 3 - 9.VII.1997: 11 , Farano; 4 - 11.XII.1997: 1 p, Farano.
Caldognola: 1 - 26.VII.1994: 1 p, Brunetti; 2 - 25.VII.1995: 1 ô, Brunetti.; 4 - 1.IX.1995: 1 क , Brunetti; 5 - 22.IX.1994: 7 1; 25.IX. 1994: 1 oै, 1 p o , 19.III.1995: 1 1; 7.V.1995: 1 1; 1.IX.1995: 1 ô, Brunetti.

Menotre: 2 - 2004-2005: 74 1, Todini; 4 - 11.V.1987: 6 1; 14.VII.1987: 1 1; 30.VII.1987: 1 1, Fortini; 5 - 11.V.1987: 6 1, Fortini; 2004-2005: 3 1, Todini; 8 - 2004-2005: 35 1, Todini; 9-11.V.1987: 1 1, Fortini.

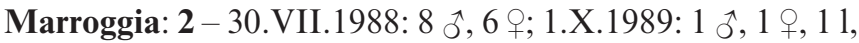
Arena; 3 - 10.V.1987: 1 ô, 1 1; 30.VII.1988: 1 ô, 1 1, Arena (Di Giovanni et al., 2004).

\section{Melampophylax melampus (McLachlan, 1876)}

Chiona: 3 - 25.X.1995: 3 ô, 1 ㅇ (L), Fucchi.

\section{Stenophylax mitis (McLachlan, 1875)}

Chiona: 1 - 30.III.1993: 1 ô, 2 o, 1 1, Cardinali; 2 - 19.IV.1993: 1 ô, 2 ㅇ, 1 1, Cardinali; 6 - 17.III.1992: 11 , Cardinali.

Sorgente Fosso delle Piagge: 30.III.1993: 1 ô, 2 क , 1 1, Cardinali.

Palude di Colfiorito: 4 - 14.III.1999: 2 ô, 2 , , Cianficconi, Mazzerioli, Salerno (Cianficconi et al., 2000).

Stenophylax mucronatus (McLachlan, 1880)

Chiona: 3 - 8.IX.1995: 2 (L), Fucchi.

Palude di Colfiorito: 4 - 14.III.1999: 2 ^ิ, 2 \&, Cianficconi, Mazzerioli, Salerno; 6 - 25.III.1996: 1 ô, 3 ㅇ, Mazzerioli, Salerno (Cianficconi et al., 2000).

\section{Stenophylax permistus (McLachlan, 1895)}

Menotre: 5 - 2004-2005: 1 1, Todini.

Palude di Colfiorito: 4 - 14.III.1999: 3 ô, 6 q, Cianficconi, Mazzerioli, Salerno (Cianficconi et al., 2000).

\section{Micropterna fissa (McLachlan, 1875)}

Menotre: 10 - 27.X.1988: 1 + , Fortini (Di Giovanni et al., 2004).

\section{Micropterna nycterobia (McLachlan, 1875)}

Topino: 01 - VII.1997: 4 1, Cappelletti; 03 - VII.1997: 20 1, Cappelletti.

\section{Micropterna sequax (McLachlan, 1875)}

Sorgente Fonte Vecchia: 28.IV.1992: 1 ^ึ, 2 क , 1 1; 5.V.1993: $2 \hat{\jmath}, 1$ p, Cardinali. 
Palude di Colfiorito: 4 - 14.III.1999: 1 ô, 1 1, Mazzerioli, Salerno (Cianficconi et al., 2000).

\section{Micropterna testacea (Gmelin, 1789)}

Chiona: 7 - 12.VI.1995: 1 क , Fucchi; 9 - 9.V.1995: 2 ^), 3 \%; 11.VI.1995: 1 , Fucchi.

Caldognola: 2 - 7.V.1995: 1 , , Brunetti.

Marroggia: 1 - 29.I.1989: 3 , Arena; 2 - 29.I.1989: 2 , 2 1; 12.XI.1990: 1 ô, 1 1, Arena (Di Giovanni et al., 2004).

\section{Mesophylax aspersus (Rambur, 1842)}

Menotre: 2 - 2004-2005: 4 1, Todini; 5 - 2004-2005: 3 1, Todini.

Lago de L'Aiso: 2 - XI.1991: 1 1, (Di Giovanni et al., 1992).

Marroggia: 3 - 2.IV.1989: 1 ^ै, 2 ค, 2 1, Arena (Di Giovanni et al., 2004).

\section{Allogamus antennatus (McLachlan, 1876)}

Menotre: 0 - 30.VII.1987: 1 1, Fortini; 1 - 28.XI.1986. 1 1; 27.X.1988: 1 क, Fortini (Di Giovanni et al., 2004); 3 - 7.XII.1986: 3 p; 12.III.1987: 1 1; 11.V.1987: 293 1, Fortini (Di Giovanni et al., 2004); 2 - 2004-2005: 22 1, Todini; 5-11.V.1987: 160 1; 20.X.1987: 1 +; 27.X.1988: 4 , Fortini (Di Giovanni et al., 2004); 2004-2005: 1 1, Todini;

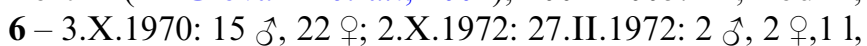
Celletti (Di Giovanni et al., 2004); 27.III.1972: 1 1; 27.IV.1972: 1 1; 27.V.1972: 1 1; 28.V.1972: 1 1; 28.VI.1972: 1 1; 27.VII.1972: 1 1; 27.VIII.1972: 1 1; 22.IX.1972: 1 1; 2.X.1972: 1 ô, Guidi (Di Giovanni et al., 2004); 7 - 13.IV.1987: 1 1; 11.V.1987: 1 1; 14.VII.1987: 4 1, Fortini; 8-2004-2005: 26 1, Todini; 9-11.V.1987: 1 1, Fortini.

\section{Allogamus ausoniae (Moretti, 1991)}

Topino: 2 -21.VII.1995: 1 ô, 2 1, Montanucci.

\section{Chaetopteryx gessneri (McLachlan, 1876)}

Topino: 01 - IV.1997: 18 1; VI.1997: 6 1; VII.1997: 26 1; X.1997: 12 1; I.1998: 35 1, Cappelletti; 02 - 6.VII.1949: 1 ô, 1 1, Moretti (Di Giovanni et al., 2004); 9.X.1950: 4 p, Moretti, Cianficconi (Di Giovanni et al., 2004); 22.XI.1950: 17 ô, 3 , Bellini (Di Giovanni et al., 2004); 9-21.1950: 1 ô, 17 1; 9.X.1952: 1 क, 49 p, 140 1, Gianformaggio (Di Giovanni et al., 2004); 03 - 22.IX.1979: 3 p; 4.XII.1979: 1 ô, 1 ㅇ, Lipparelli (Di Giovanni et al., 2004); VI.1997: 16 1; VII.1997: 40 1; X.1997: 36 1, Cappelletti; 1 - 9.X.1994: 11 , Montanucci; 4 - 20.X.1987: 2 ऽ, 1 †, Cianficconi, Chiappafreddo (Di Giovanni et al., 2004); 5-9.X.1994 1 ડै, Montanucci.
Fergia: 1 - 3.XI.1992: 6 ^ิ, 1 ค, Salerno (Di Giovanni et al., 2004); 5.V.1997: 1 1; 23.VII.1997: 2 1, Farano; 2 - 13.VI.1997: 4 1; 9.VII.1997: 1 1; 3 - 13.VI.1997: 1 1; 9.VII.1997: 11, Farano.

Caldognola: 3 - 11.X.1994: 1 , Brunetti.

Menotre: 1 - 3.XI.1987: 3 ô, 2 +, Cianficconi, Chiappafreddo, Fortini, (Di Giovanni et al., 2004); 4 - 20.X.1987: 2 ô, 1 क, Cianficconi, Chiappafreddo, Fortini, (Di Giovanni et al., 2004); 5 - 20.X.1987: 1 ô, Cianficconi, Chiappafreddo, Fortini (Di Giovanni et al., 2004).

Clitunno: 1 - 30.XI.1964: 1 + ; 9.X.1970: 1 ڤึ; 19.XI.1972: $2 \hat{o}, 1$ ㅇ, Moretti; 3.I.1970: 1 +, Pirisinu (Di Giovanni et al., 2004); 9-18.XI.1970: 1 ô, 1 ㅇ; 22.I.1972 1 ô, 1 ㅇ, Sbrenna (Di Giovanni et al., 2004); 15.X.1972: 1 ô, Corvelli.

\section{Goeridae}

\section{Silo mediterraneus (McLachlan, 1884)}

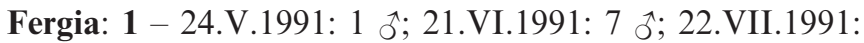
1 ơ; 1.VIII.1991: 1 ô, Salerno (Di Giovanni et al., 2004); 21.I.1997: 1 1; 5.V.1997: 1 ô, 1 p, 1 1; 9.VII.1997: 1 p; 23.VII.1997: 5 ^ै, 1 p; 2 - 26.X.1996: 1 1; 5.V.1997: 1 ; 13.VI.1997: 5 ơ; 9.VII.1997: 1 p, 1 1, Farano.

Menotre: 2 - 2004-2005: 201 Todini; 3 - 27.III.1972: 1 p; 27.IV.1972: 1 1; 28.VI.1972: 1 1, Guidi; 30.VII.1987: 3 ô., Cianficconi, Chiappafreddo, Fortini (Di Giovanni et al., 2004); 18.V.1988: 6 ô, 1 क, Cianficconi, Chiappafreddo, Fortini (Di Giovanni et al., 2004); 4 - 7.XII.1986: 2 oै, 1 क: 12.III.1987: 1 ^ै, 3 क; 13.IV.1987: 4 क; 11.V.1987: 2 ô, 2 क; 16.VI.1987: 3 ô, 1 क; 30.VII. 1987: 3 ô, Fortini (Di Giovanni et al., 2004); 27.IV.1972: 1 1; 27.VIII.1972: 1 p, Guidi; 5 - 11.V.1987: 2 ; 16.VI.1987: 2 ô; 20.VII.1988: 1 ô, Fortini (Di Giovanni et al., 2004); 7.XII.1986: 2 ô, 1 o, Cianficconi, Chiappafreddo, Fortini (Di Giovanni et al., 2004); 18.V.1988: 1 ô, 1 ㅇ, Cianficconi, Chiappafreddo, Fortini (Di Giovanni et al., 2004); 20.VII.1988: 1 ô, Moretti, Cianficconi, Chiappafreddo, Fortini (Di Giovanni et al., 2004); 2004-2005: 2 1, Todini; 6 - 27.III.1972: 1 p; 27.IV.1972: 1 1, Guidi; 7 - 11.V.1987: 1 ô, 1 +, Fortini (Di Giovanni et al., 2004).

Clitunno: 1 - 9.V.1948: 1 ๙ै, 77 p, 6 1; 31.III.1952: 136 ô, 11

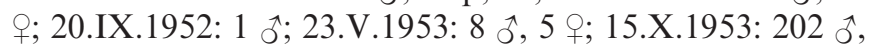
12 \%; 11.V.1956: 15 ô, 7 क; 21.IV.1967: 1 ऊิ; 27.V.1967: 35 ô, 11 क , Moretti (Di Giovanni et al., 2004); 19.IV.1955: 174 ô, 83 क; 15.X.1958: 37 ๙ै, 12, Bellini (Di Giovanni et al., 2004); 31.III.1952: 92 p, 8 1; 3.VI.1952: 93 p, 41 1; 6.X.1952: 12 p; 23.V.1953: 7 p; 24.VII.1953: 5 p; 20.IX.1953: 129 p, 14 1, Casagrande (Di Giovanni et al., 2004); 23.IV.1967: 3 p, Griselli (Di Giovanni et al., 2004);

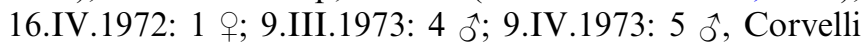
(Di Giovanni et al., 2004); 18.IX.1972: 3 क; 15.X.1972:

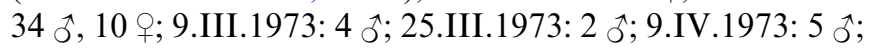




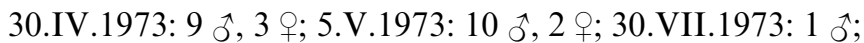
6.X.1973: 29 ô, 7 o, Moretti.

Marroggia: 1 - 8.III.1987: 2 ๙ , 4 +, 3 1; 12.IV.1987: 2 क, 2 1;

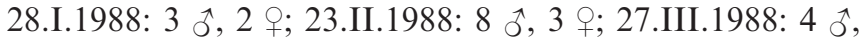
2 क; 29.I.1989: 4 คै, 2 क; 2.IV.1989: 3 ô, 2 +, 2 1; 7.V.1989:

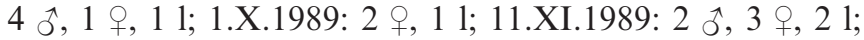
24.III.1991: 51 ô, Arena (Di Giovanni et al., 2004); 2 -26.IV.1988: 9 ô; 24.V.1988: 3 ô, 6 o; 14.XI.1988: 1 \%, 1 1; 29.I.1989: 4 o, 3 1; 6.III.1989: 1 ô, 3 o, 3 1; 2.IV.1989:

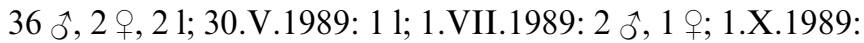
17 oै, 18 क , 6 1; 11.XI.1989: 27 ô, 11 ㅇ, 2 1; 21.I.1990: 1 oै, 1 1; 29.IV.1990: 8 ô, 23 क, 4 1; 4.VI.1990: 3 क, 1 1; 12.XI.1990: 1 q, 1 1; 27.I.1991: 1 +, 1 1; 24.III.1991: 3 ô, Arena (Di Giovanni et al., 2004); 3 - 9.III.1987: 1 ô, 1 1; 10.V.1987: 1 今ૈ, 1 1; 23.II.1988: 1 ô, 1 1; 29.IV.1990: 1 +, 1 1, Arena (Di Giovanni et al., 2004).

\section{Lepidostomatidae}

\section{Lepidostoma basalis (Kolenati, 1848)}

Topino: 03-2.VII. 1979: 2 $\widehat{\jmath}, 10$ ㅇ, Lipparelli (Di Giovanni et al., 2004).

Menotre: 1 - 6-15.VI.1979: 3 ô, 10 1, Orecchini (Di Giovanni et al., 2004); 2 - 2004-2005: 5 1, Todini; 4 - 7.XII.1986: 3 क ; 12.III.1987: 3 ^ै, 3 क; 13.IV.1987: 1 \%; 11.V.1987: 2 ㅇ, 86 1; 14.VII.1987: 6 ô, 1 1, Fortini (Di Giovanni et al., 2004); 5 - 7.XII.1986: 1 रे;

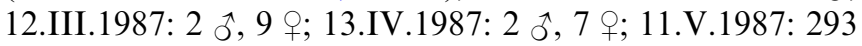

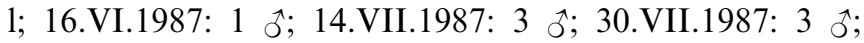
20.VII.1988: 11 ^ิ, 11 क , Fortini (Di Giovanni et al., 2004); 7 - 19.XII.1986: 2 今ै; 12.III.1987: 1 ô, 1 क; 11.V.1987: 1 1; 16.VI.1987: 2 oै, 2 क; 14.VII.1987: 2 今ै; 30.VII.1987: 1 ○ै, Fortini (Di Giovanni et al., 2004); 8-16.VI.1985: 76 ô, 1 +, Moretti, Tucciarelli, Chiappafreddo (Di Giovanni et al., 2004); 9-11.V.1987: 1 1, Fortini (Di Giovanni et al., 2004).

\section{Lepidostoma hirtum (Fabricius, 1775)}

Menotre: 5 - 30.IV.1972: 3 ô; 10,13,22.V.1972: 3 ô, 3 क, Sbrenna; 20.VII.1988: 3 +, Moretti, Cianficconi Chiappafreddo, Fortini (Di Giovanni et al., 2004).

\section{Crunoecia irrorata (Curtis, 1834)}

Topino: 01 - 19.III.1975: 1 ㅇ, Lipparelli (Di Giovanni et al., 2004).

Chiona: 9 - 10.IV.1996: 1 1, Fucchi.

Fergia: 1 - 23.VII.1997: 4 ô, 1 क, 1 p, 18 1, Farano; 2-13.VI.1997: 1 q Farano.

Menotre: 0 - 30.VII.1987: 1 ô, Cianficconi, Chiappafreddo, Fortini (Di Giovanni et al., 2004).

\section{Leptoceridae}

Mystacides azurea (Linnaeus, 1761)

Palude di Colfiorito: 2 - 7.IX.1993: 1 ô(L), Salerno, Tavone; 29.VI.1994: 1 우 (L), Cianficconi, Mazzerioli, Moretti, Salerno, Speziale (Cianficconi et al., 2000); 9 - 10.VII.1998: 2 ô, 2 o, Mazzerioli, Salerno (Cianficconi et al., 2000).

\section{Erotesis baltica (McLachlan, 1877)}

Clitunno: 1 - 3.VI.1952: 3 कै; 5.VIII.1952: 1 ऊิ; Moretti (Di Giovanni et al., 2004); 3.VI.1979: 1 ㅇ, Moretti, Cianficconi, Marini (Di Giovanni et al., 2004).

\section{Leptocerus tineiformis (Curtis, 1834)}

Palude di Colfiorito: 2 - 29.VI.1994: 1 ^ (L), Cianficconi, Mazzerioli, Moretti, Salerno, Speziale (Cianficconi et al., 2000); 6.VIII.1996: 1 क (L), Mazzerioli, Salerno (Cianficconi et al., 2000); 5 - 10.VI.1998: 2 \& (L), Mazzerioli, Salerno (Cianficconi et al., 2000).

\section{Sericostomatidae}

\section{Sericostoma cianficconii (Moretti, 1978)}

Menotre: 2 - 2004-2005: 11 1, Todini; 4 - 12.III.1987: 1 गै, 1 \%; 11.V.1987: 2 \&, Fortini (Di Giovanni et al., 2004), 30.VII.1987: 1 ô, Cianficconi, Chiappafreddo, Fortini (Di Giovanni et al., 2004); 5-5-15.VI.1972: 15 ^, 10 o , Sbrenna (Di Giovanni et al., 2004); 12.III.1987: 3 ô; 13.IV.1987: 3 कें; 11.V.1987: 13 1, Fortini (Di Giovanni et al., 2004); 20.VII.1988: 1 ๙ै, 2 \%, Moretti, Cianficconi, Chiappafreddo (Di Giovanni et al., 2004); 7 - 12.III.1987: 1 +; 11.V.1987: 1 1; 14.VII.1987: 1 1, Fortini (Di Giovanni et al., 2004).

\section{Sericostoma pedemontanum (McLachlan, 1876)}

Fergia: 1 - 22.VII.1991: 1 ô, 5 op; 1.VIII.1991: 5 ô; 21.VIII.1991: 3 oै; 15.X.1992: 10 क; 3.XI.1992: 1 ô, Salerno (Di Giovanni et al., 2004); 21.I.1997: 1 1; 5.V. 1997: 1 p, 1 1; 23.VII.1997: 1 p, Farano; 2 - 26.X.1996: 1 ㅇ, 3 1; 13.VI.1997: 1 क, 3 1; 23.VII.1997: 3 ô, 3 क; 3 - 09.VII.1997: 2 1; 5 - 16.IX.1997: 1 ô Farano; 6 - 13.VIII.1991: 5 ô, 4 ㅇ Salerno (Di Giovanni et al., 2004).

Caldognola: 4 - 26.VII.1994: 1 ๙

Fonte Orbi: 16.IV.1992: 2 ㅇ, 8.V.1993: 1今̂, 3 ㅇ, 11 Cardinali.

Sorgente del Fosso Trosceto: 12.V.1992: $2 \hat{\jmath}, 1$ क , 1 p, 8.VII.1992: 20ิ, 1 क, 24.X.1992: 1 1, 22.III.1993: 1 1, 11.IV.1993: 20ิ, 1 \& Cardinali. 
Fonte S. Giovanni: 16.IV.1992: 1 ^ึ, 2 \&, 1 1, 8.V.1993: 11 Cardinali.

Menotre: 0 - 30.VII.1987: 1 ô, 1 Moretti, Cianficconi, Chiappafreddo, Fortini (Di Giovanni et al., 2004); 1 - 30.VII.1987: 2 ô, 1 \& Cianficconi, Chiappafreddo Fortini, 04.VII.1989 (Di Giovanni et al., 2004): 2 ๙ Moretti, Cianficconi, Chiappafreddo (Di Giovanni et al., 2004); 2 - 20.VII.1988: 3 ô, 1 † Cianficconi, Chiappafreddo, Fortini (Di Giovanni et al., 2004); 20042005: 521 Todini; 5 - 20.VII.1988: 1 क Cianficconi, Chiappafreddo, Fortini (Di Giovanni et al., 2004); 20042005: 131 Todini; 9 - 13.IV.1987: 1 ô, 11.V.1987: 1 1, 14.VII.1987: 11 Fortini.

Clitunno: 1 - 03.VI.1952: 1 ô, 24.VII.1953: 2 ô, 3 के, 15.X.1953: 2 o, 2 ㅇ Moretti, Bellini (Di Giovanni et al., 2004); 19.IV.1955: 2 ô Bellini (Di Giovanni et al., 2004); 20.V.1958: 1 क Giannotti, 28.VI.1964: 1 क T Tristaino, 05.V.1973: 1 ऽิ, 1 ㅇ, 6.X.1973: 1 우, 21.X.1973: 1 ㅇ, 15.X.1983: 2 + Moretti.

Marroggia: 1 - 12.IV.1987: 1 个, 1 1, 21.VIII.1987: 5 ^ิ, 3 q, 24.XI.1987: 3 ô, 2 क , 4 1, 27.III.1988: 1 ô, 1 ㅇ, 24.V.1988: 1 o, 14.XI.1988: 1 ㅇ, 29.I.1989: 1 o, 1 1, 6.III.1989: 1 o, 11 , 2.IV.1989: 1 ô, 1 क , 7.V.1989: 3 ô, 5 o +, 1 1, 1.VII.1989: 1 ô, 1 ㅇ, 1 1, 01.X.1989: 1 1, 1 +, 11.XI.1989: 21,1 ô, 1 \%; 2 - 29.I.1989: 1 ô, 2 क, 2 1, 06.III.1989: 1 ô, 3 क, 3 1, 02.IV.1989: 2 ô, 1 1, 30.V.1989: 1 ô Arena (Di Giovanni et al., 2004).

\section{Beraeidae}

\section{Beraea maurus (Curtis, 1834)}

Fergia: 3 - 13.VI.1997: 2 ô, 1 \%; 4 - 16.IX.1997: 11 Farano.

Clitunno: 1 - 03.VI.1952: 1 ô, 23.V.1953: 2 ô, 1 ㅇ, 25.V.1953: 2 ô, 1 + Casagrande (Di Giovanni et al.,

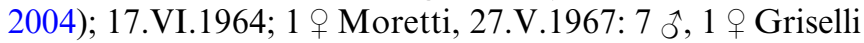
(Di Giovanni et al., 2004); 18.IX.1972: 1 ô, 30.IV.1973: 1 ô Corvelli.

\section{Beraeodes minutus (Linnaeus, 1761)}

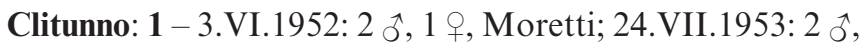
Bellini; 30.IV.1973: 1 ๙ै, Corvelli, 1.VI.1979: 551 ô, 107 ㅇ, Moretti, Cianficconi, Marini (Di Giovanni et al., 2004); 27.V.1981: 16 p, 19 1, Bicchierai, Marini, Chiappafreddo (Di Giovanni et al., 2004); 14.VI.1981: 288 ô, 54 우 Bicchierai, Marini, Chiappafreddo (Di Giovanni et al., 2004).

\section{Beraeamyia squamosa (Mosely, 1930)}

Marroggia: 3 - 30.V.1989: $1 \mathrm{p}$ ô, $1 \mathrm{p}$ ㅇ, Arena (Di Giovanni et al., 2004).

\section{Odontoceridae}

\section{Odontocerum albicorne (Scopoli, 1769)}

Topino: 01 - 9-19.III.1979: 1 ô, Lipparelli (Di Giovanni et al., 2004); 03 - VI.1997: 4 1, Cappelletti; 1 - 9.X.1994: 1 1; 29.XI.1994: 6 1; 3.VI.1995: 1 oै, 1 क, 9 p, 3 1; 21.VII.1995: 10 p, 2 1; 20.III.1996: 11 1, Montanucci; 2 - 18.III.1995: 1 1; 21.V.1995: 2 ô, 4 1; 21.VII.1995: 3 p; 20.III.1996: 7 1, Montanucci; 3 - 8.X.1994: 5 1; 3.VI. 1995: 4 ๙ै, 1 ㅇ, 16 p, 8 1; 20.III.1996: 5 1, Montanucci; 4 - 12.III.1995: 1 p; 21.V.1995 1 ô, 12 क; 21.VII.1995: 2 p o; 23.VIII.1995: 5 + 1 p; 20.III.1996: 61, Montanucci; 5 - 7.IX.1994: 2 ô, 1 ㅇ; 22.IX.1994: 6 p, 4 1; 30.IV.1995: 3 1; 21.VII.1995: 11 क, 5 1; 20.III.1996: 5 1; 21.VII.1995: 1 , 1 p, 71 , Montanucci.

Fergia: 1 - 22.VII.1991: 2 \%; 13-21.VIII.1991: 29 ㅅ, 10 \%; 18.IX.1992: 7 क , Salerno (Di Giovanni et al., 2004); 21.I.1997: 1 ô, 1 p, 1 1; 4.III.1997: 1 p, 1 1; 9.VII.1997: 1 1; 23.VII.1997: 1 ô, 2 क , 1 p, 1 1; 2 - 13.VI.1997: 1 1; 9.VII.1997: 1 p, 1 1; 23.VII.1997: 8 ô, 2 क, Farano; 3 - 13.VI.1997: 4 +, 1 p; 9.VII.1997: 1 ô, 1 p; 16.IX. 1997: 1 ô, 1 p, Farano; 5 - 16.IX.1997: 1 ô, Farano; 6 - 13.VI.1997: 1 ô Farano.

Caldognola: 3 - 5.VII.1995: 1 p, Brunetti; 4 - 26.VII.1994: 7 ô, 57 ㅇ (L), Brunetti; 5 - 22.IX.1994: 2 1; 18.VII.1995: 91 , Brunetti.

Chiona: 3 - 9.VIII.1995: 1 ^ै, 43 q (L); 11.VI.1996 2 1; 16.IX.1996: 2 1, Fucchi; 5 - 31.VII.1995: 3 ô, 1 ㅇ (L), Fucchi.

Menotre: 0 - 30.VII.1987: 1 ô, Cianficconi, Chiappafreddo, Fortini (Di Giovanni et al., 2004); 1 - 11.V.1987: 1 1; 30.VII.1987: 5 ô, 3 o, Cianficconi, Chiappafreddo, Fortini (Di Giovanni et al., 2004); 27.VII.1972: 1 1, Guidi; 3.VI.1978: 1 ô, Orecchini (Di Giovanni et al., 2004); 2 - 2004-2005: 43 1, Todini; 3 - 27.VIII.1972: 1 1, Guidi; 4 - 7.XII.1986: 1 ; 12.III.1987: 1 ㅇ; 30.VII.1987: 1 ภิ, 2 ㅇ, Cianficconi, Chiappafreddo, Fortini (Di Giovanni et al., 2004); 27.X.1971: 1 1; 27.XI.1971: 1 1; 27.I.1972: 1 1; 27.IV.1972: 1 1; 28.V.1972: 1 1; 27.VIII.1972: 1 1, Guidi; 5-2004-2005: 24 1, Todini; 7 - 12.III.1987: 1 1; 11.V.1987: 1 1, Fortini; 8-2004-2005: 12 1, Todini.

\section{Discussion and conclusion}

During the research carried out from 1947 to 2005 at 61 sampling sites and 51 habitat types, 6012 adults (3484 $\hat{~}$. and 2528 \%) and numerous aquatic stages were collected. In total, 83 taxa (78 species and 5 subspecies) were recorded (Table 1), including 16 species and 5 subspecies endemic to the Italian fauna. This inventory represents $18 \%$ of the species and $18.7 \%$ of the subspecies of the Italian Trichopteran fauna (416 species and 32 subspecies), that belong to 41 genera and 17 families of the 93 genera and 20 families known in Italy. 


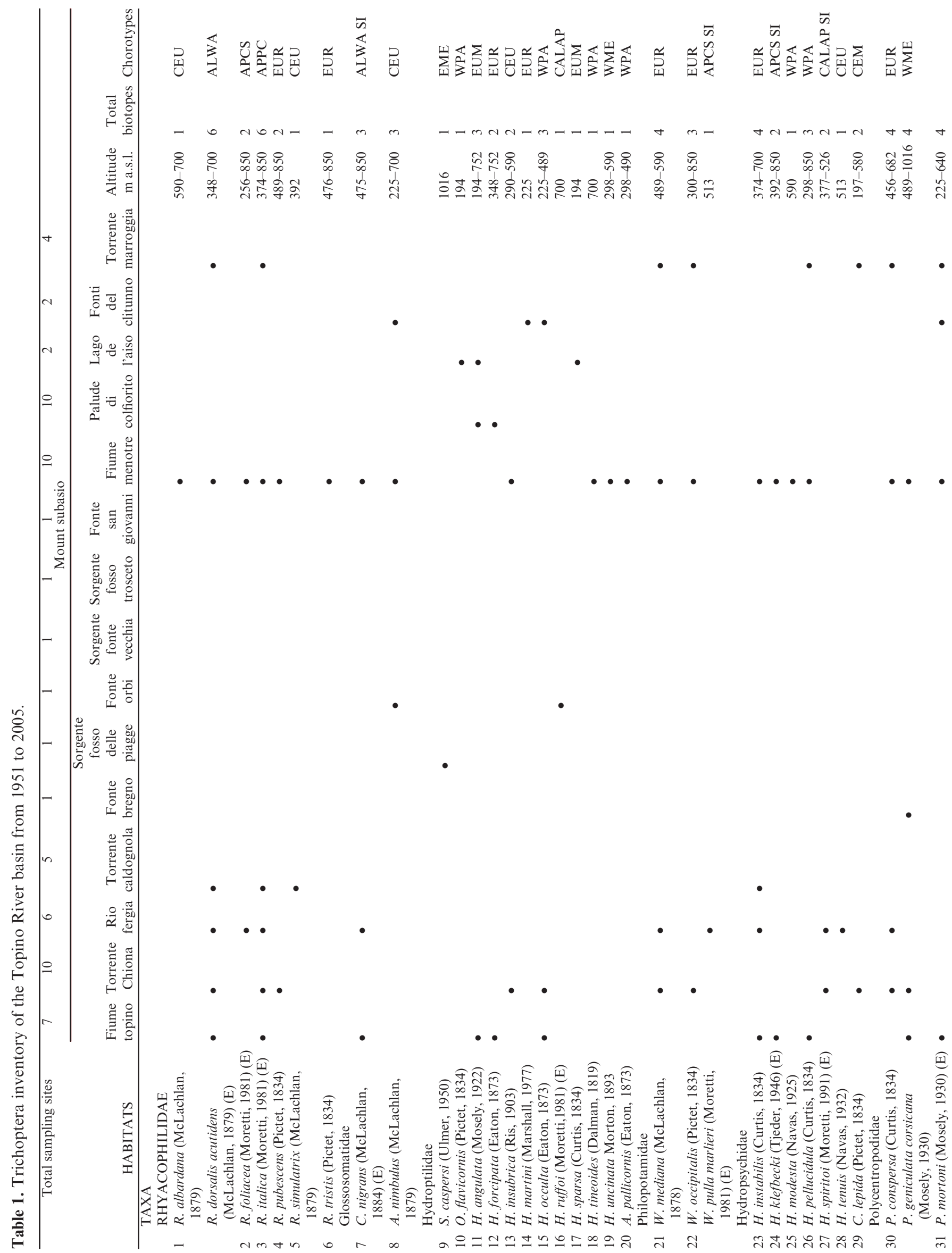




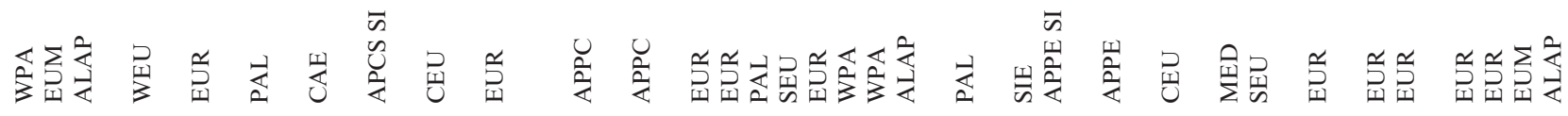
- n. . - . . - -

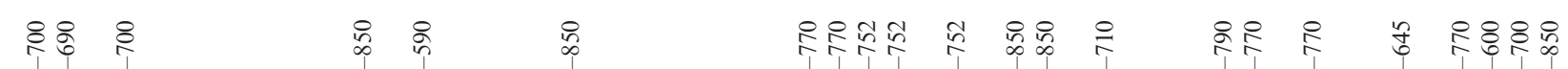

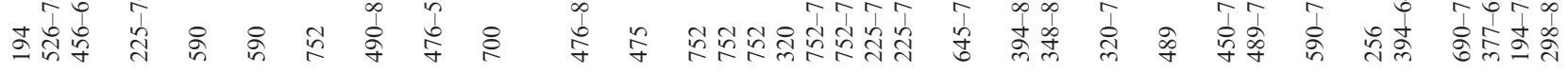
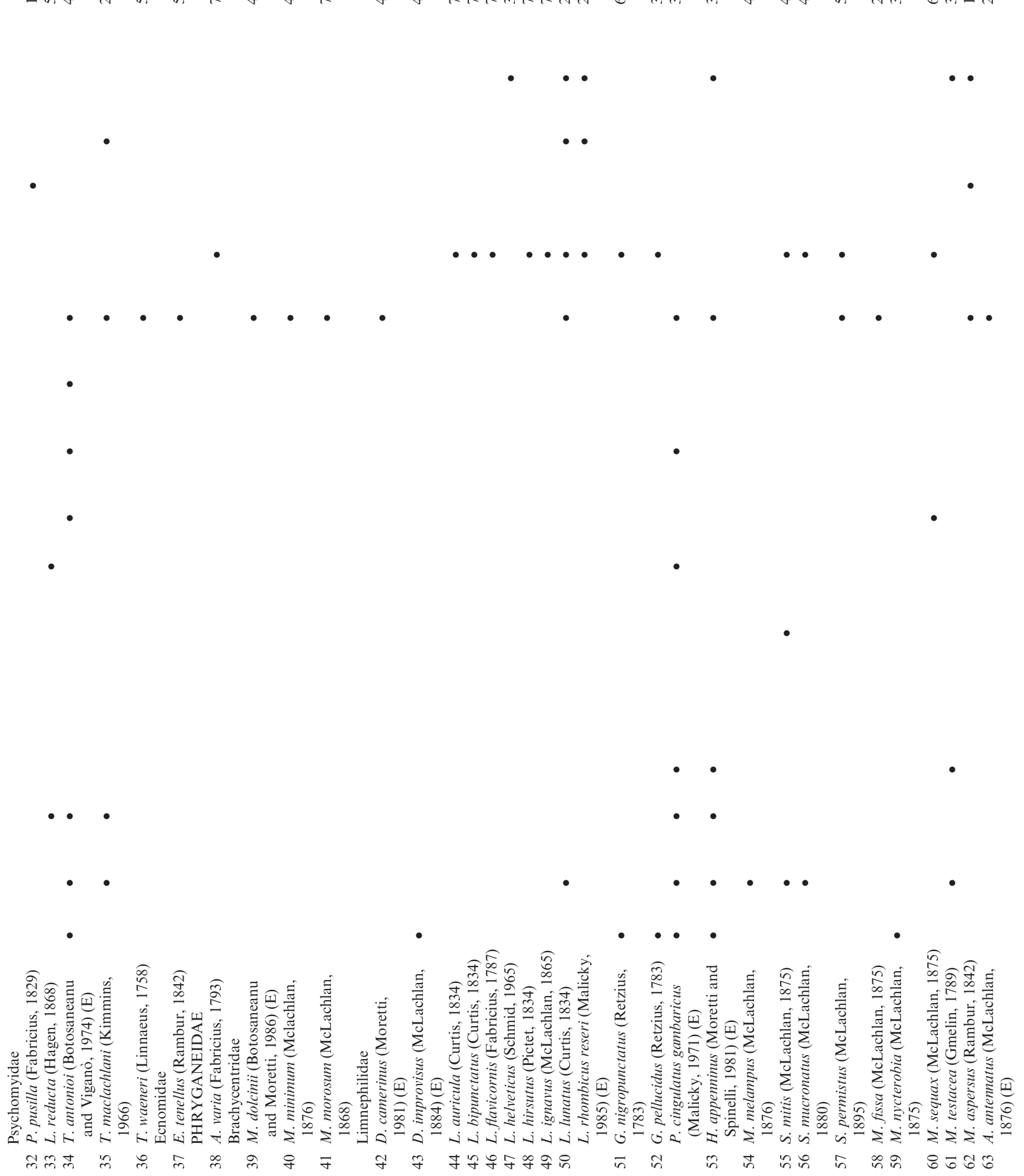


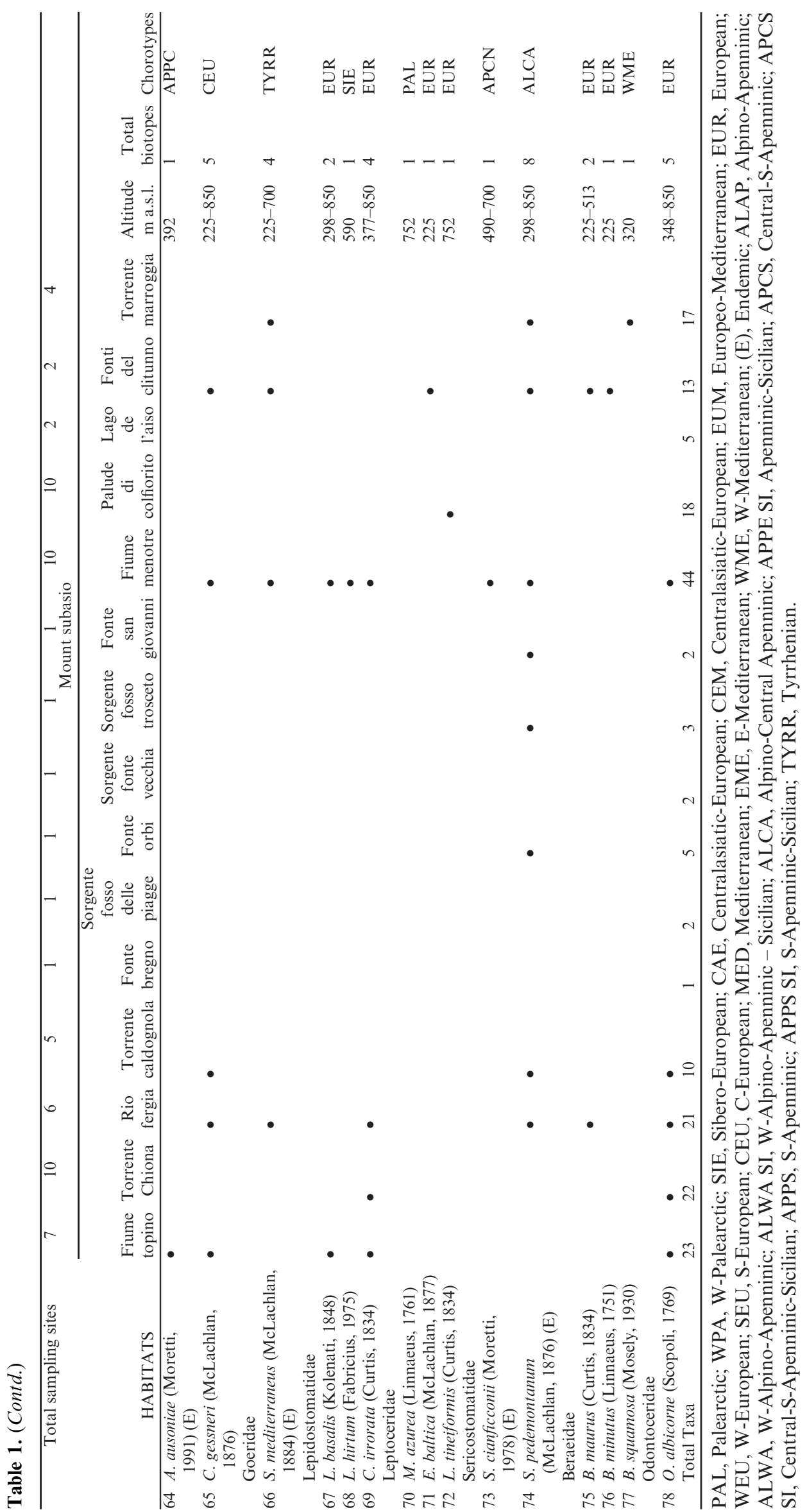




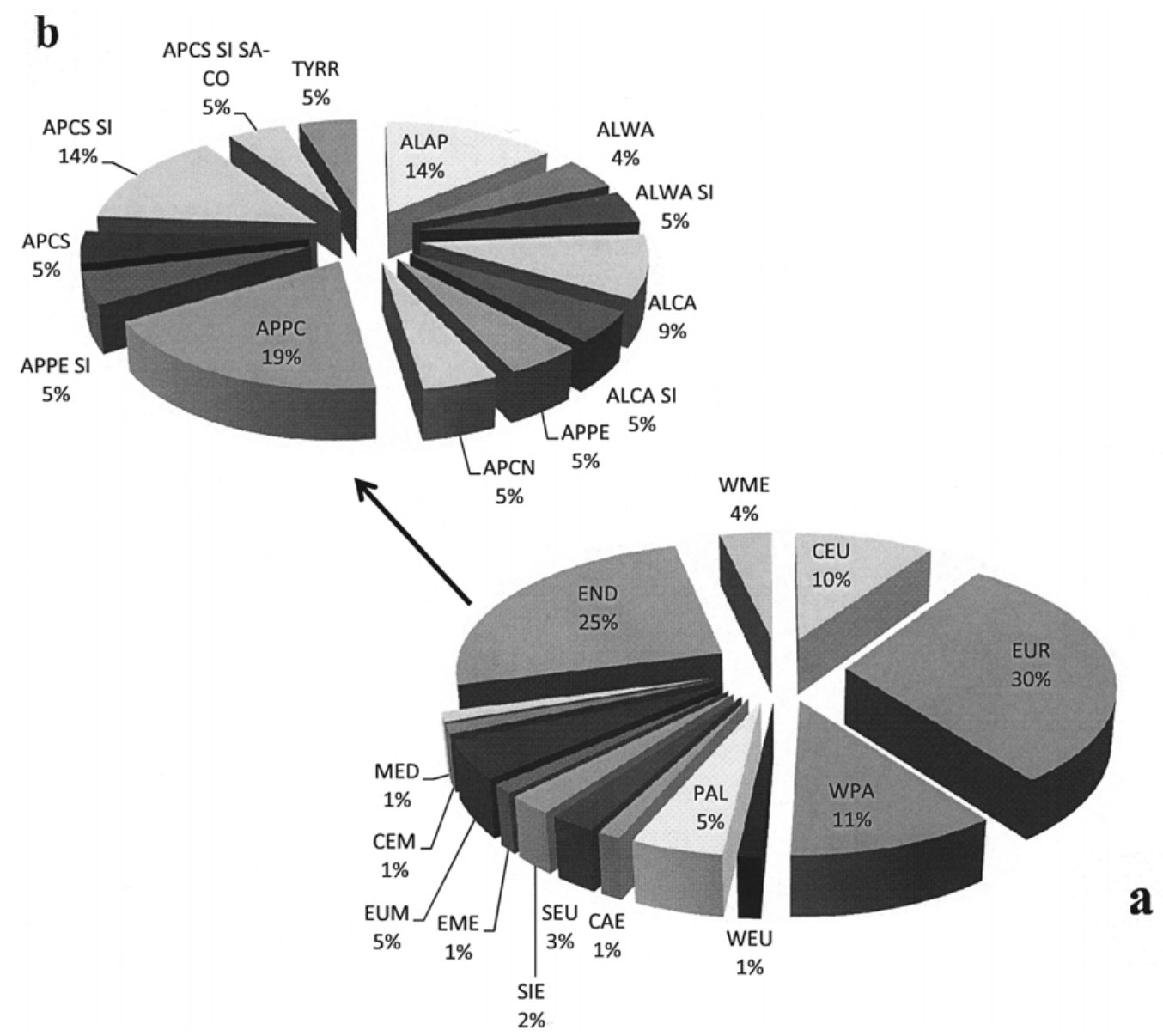

Fig. 6. Large-scale geographical distribution patterns (Vigna Taglianti et al., 1999) of the Topino basin Trichoptera species and subspecies (lower section of figure, (a) and distribution of taxa endemic to Italy (END, upper section of figure, (b). a: PAL, Palearctic; WPA, W-Palearctic; SIE, Sibero-European; CAE, Centralasiatic-European; CEM, Centralasiatic-European; EUM, EuropeoMediterranean; EUR, European; WEU, W-European; SEU, S-European; CEU, C-European; MED, Mediterranean; EME, E-Mediterranean; WME, W-Mediterranean; End, Endemics. b: ALAP, Alpino-Apenninic; ALWA, W-Alpino-Apenninic; ALWA SI, W-Alpino-Apenninic-Sicilian; ALCA, Alpino-Central Apenninic; APPE SI, Apenninic Sicilian; APCS, Central-S-Apenninic; APCS SI, Central-S-Apenninic-Sicilian; APPS, S-Apenninic; APPS SI, S-Apenninic-Sicilian; TYRR, Tyrrhenian.

The families with the highest number of species are Rhyacophilidae with 6 species and 1 subspecies of Rhyacophila out of the 35 species and 4 subspecies present in Italy, Limnephilidae with 7 species and 1 subspecies of Limnephilus out of the 29 species and 1 subspecies present in Italy, 3 species of Stenophylax of the 6 species present in Italy, 4 species of Micropterna of the 8 species present in Italy and Hydroptilidae with 9 species of the 34 species of Hydroptila present in Italy.

The most widespread species include $S$. pedemontanum present at eight habitats, $T$. antonioi and $P$. cingulatus gambaricus (seven habitats), Halesus appenninus, $R$. dorsalis acutidens and $R$. italica found in six habitats.

With respect to ecology of the species, our inventory comprises euryoecious (e.g., H. pellucidula, $R$. dorsalis acutidens) as well as stenoecious species, e.g., madicole (B. maurus, T. maclachlani), crenobiont (Catagapetus nigrans, Drusus cameriunus, D. improvisus, Chaetopteryx gessneri, Crunoecia irrorata), crenoxene (S. mediterraneus,
S. pedemontanum, Odontocerum albicorne) and epirithral species which are the most numerous.

In lentic waters we recorded various species of Limnephilidae, 12 of them in the Palude di Colfiorito. Seven of these spcies belong to the genus Limnephilus: L. auricula and L. ignavus are present in Umbria only at this site. Rare species were recorded in the Fonti del Clitunno (E. baltica and Beraeodes minutus; Fig. 5) and in the Topino River (Wormaldia pulla marlieri). The River Menotre is the richest system investigated (44 species including 9 endemics), followed by the Topino River with 23 species (including 8 endemics) and the Chiona River with 22 species (including 6 endemics). The elevation of the sampling sites ranges from $197 \mathrm{~m}$ (T. Chiona) to $1016 \mathrm{~m}$ a.s.l. (Fonte Bregno) for lotic waters sites and from $194 \mathrm{~m}$ (Lago dell'Aiso) to $770 \mathrm{~m}$ a.s.l (Palude di Colfiorito) for lentic waters sites.

With respect to geographical distribution patterns (Table 1, Fig. 6), 44\% of the species are restricted to Europe, $24 \%$ are widely distributed (Palearctic, W-Palearctic, 
Sibero-European, Centralasiatic-European, CentralasiaticEuropeo-Mediterranean, Europeo-Mediterranean) and $6 \%$ are restricted to the Mediterranean. In total, $25 \%$ of the species are endemic to the Italian fauna, e.g., Apenninic ( $R$. dorsalis acutidens, $S$. pedemontanum), Central-S-Apenninic ( $R$. foliacea, Hydropsyche spiritoi) and Central-Apenninic ( $R$. italica, Wormaldia mediana, W. pulla marlieri, D. camerinus, D. improvisus, Halesus appenninus, $A$. ausoniae).

Based on our data the Trichoptera fauna of the Topino basin is of great systematic, ecological and chorological interest when compared with inventories of the nine other drainage basins in the region of Umbria. The numerous tectonic phenomena in central Italy from about 5.33 million years ago have played a fundamental role in shaping the geology and the variety of environments in the River Topino basin ranging from rheocrenous and limnocrenous springs to a variety of running and lentic waters. The numerous springs within the study area support a high Trichoptera diversity, and deserve particular attention within the framework of environmental management plans.

Acknowledgements. We would like to thank Professor Roberto Colacicchi for the invaluable help that he gave us in reviewing the geological data of this paper.

\section{References}

Arena C. 1990-1991. Tricotterofauna di un torrente della piana spoletina (T. Marroggia). Scienze Biologiche (Relatore: F. Cianficconi).

Bizzarri R., Albianelli A., Argenti P., Baldanza A., Colacicchi R. and Napoleone G., 2011. The latest continental filling of Valle Umbra (Tiber Basin, central Italy) dated to one million years ago by magnetostratigraphy. Il Quaternario, 24, 51-65.

Brunetti C., 1994-95. La tricotterofauna del T. Caldognola, tributario di destra del F. Topino (Umbria: PG). Scienze Naturali (Relatore: F. Cianficconi).

Capellini G., 1888. Sui resti di Mastodon arvernensis recentemente scoperti a Spoleto, Pontremoli e Castrocaro. Memorie della Regia Accademia delle Scienze, 4/9, 251-258.

Cappelletti C. 1997-98. Studio delle sorgenti di Nocera Umbra e analisi biologiche dei Tricotteri presenti in vene minori del Fiume Topino. Scienze Biologiche (Relatore C. Corallini).

Cappuccino F., Argenti P. and Baldanza A., 2006. La fauna a mammiferi della Collezione Toni: i mastodonti. In: Mattioli B. (ed.), Miniere di lignite in Umbria. Quaderni del Laboratorio di Scienze della Terra, 2-3, 24-34, GraficArte Severini, Spoleto.

Cardinali D., 1992-93. Nuove ricerche sui Tricotteri fonticoli del Massiccio del Subasio. Scienze Biologiche (Relatore: F. Cianficconi).

Cattuto C., Cencetti C. and Gregori L., 1995. Guida all'escursione Perugia-Spoleto-Todi-Orvieto. Perugia 27-29 Aprile, 1995, 16p.

Cianficconi F., 2006. Insecta Trichoptera. In: Ruffo S. and Stoch F. (eds.), Checklist and distribution of the Italian fauna. Memorie del Museo Civico di Storia Naturale di
Verona, Verona, 2 serie, Sezione Scienze della Vita, Vol. 16, 251-253 with data on CD-ROM.

Cianficconi F., Corallini C., Moretti G.P. and Salerno P. 1994. Popolamento epigeo, ipogeo e simbionti dei Tricotteri dell'Appennino umbro-marchigiano. Biogeographia, 17, 183-241.

Cianficconi F., Corallini C. and Moretti G.P., 1998. Trichopteran fauna of the Italian springs. In: Botosaneanu L. (ed.), Studies in Crenobiology. Backhuys Publishers, Leiden: 125-140.

Cianficconi F., Corallini C., Mazzerioli S. and Salerno P., 2000. Palude di Colfiorito (Umbria, Perugia): i Tricotteri e i loro simbionti. Boll. Assoc. Romana Entomol., 55, 45-66.

Cianficconi F., Salerno P. and Tucciarelli F., 2006. Elenco faunistico dei Tricotteri del Massiccio del Monte Subasio (Umbria: Perugia). Boll. Assoc. Romana Entomol., 61, $29-51$.

Cianficconi F., Tucciarelli F. and Todini B., 2007. Aggiornamento della Tricotterofauna della regione Umbria. Biogeographia, 28, 561-586.

Colacicchi R., 2010. Lineamenti geologici dell'Umbria centrale, pp.129-176, in "Invito al Parco, Invito all' Unità della Terra Lungo i Percorsi delle Sue Acque e della sua Storia" a cura di M.R. Trabalza e R. Colacicchi, Edizioni dell'Arquata, I ediz. 2007, II ediz. arricchita 2010, p. 580.

Colacicchi R. and Bizzarri R., 2008. Correlation between environmental evolution, historical settlement and cultural heritage upgrading in Valle Umbra (Central Italy). Geografia Fisica e Dinamica del Quaternario, 31, 107-118.

Coltorti M. and Pieruccini P., 1997. The southeastern Tiber Basin (Spoleto, Central Italy): geology and stratigraphy of Plio-Pleistocene sediments. Il Quaternario, 10, 159-180.

Corvelli D. 1972-73. Popolamento a tricotteri e coleotteri palpicorni delle fonti del Clitunno (Relatore: Q. Pirisinu).

Desplanques H., 1969. Campagnes ombriennes; contribution à l'étude des paysages ruraux en Italie centrale. Colin, Paris, $573 \mathrm{p}$.

Di Giovanni M.V., Goretti E., Tamanti V., Le Donne R. and Motti A., 1992. Studio limnologico di un biotopo sorgivo: il lago de L'Aiso. Riv. Idrobiol., 31, 33-49.

Di Giovanni M.V., Goretti E., Ceccagnoli D., Marcucci C. and Gianotti F.S., 2004. I Tricotteri italiani della Collezione G.P.Moretti. Catalogo aggiornato al 31/12/2003 (2002). Riv. Idrobiol., 41, 533.

Esu D. and Girotti O., 1974. La malacofauna continentale del Plio-Pleistocene dell'Italia centrale. Geol. Romana, 13, 203-293.

Esu D. and Girotti O., 1991. Late Pliocene and Pleistocene assemblages of continental molluscs in Italy. A survey. Il Quaternario, 4, 137-150.

Farano G., 1996-97. Tricotterofauna di un affluente del T. Caldognola: Rio Fergia (Umbria: PG). Scienze Naturali (Relatore: F. Cianficconi).

Fortini S., 1987-88. Un corso d'acqua umbro ricco di Tricotteri endemici: il Fiume Menotre. Scienze Biologiche (Relatore: F. Cianficconi).

Fucchi S., 1995-96. Popolamento tricotterologico di un piccolo corso d'acqua del M. Subasio: T. Chiona (Umbria, PG). Scienze Naturali (Relatore: F. Cianficconi).

Gianformaggio M., 1949-50. I fattori ecologici che regolano la vita nelle sorgenti del Fiume Topino - Nocera Umbra. Scienze Naturali (Relatore: G.P. Moretti). 
Guidi F., 1971-72. Zonazione tricotterologica nel Fiume Menotre (Foligno). Scienze Naturali (Relatore: G. Moretti).

Illies J. and Botosaneanu L., 1963. Problèmes et méthodes de la classification et de la zonation écologique des eaux courantes, considérées surtout du point de vue faunistique. Mitteilungen Internationale Vereiningung fuer Theoretische und Angewandte Limnologie, 12, 1-57.

Lipparelli N. 1978-79. Faunula tricotterologica di tre sorgenti appenniniche nel territorio di Nocera Umbra. Scienze Biologiche (Relatore: G.P. Moretti).

Lippi Boncampi C., 1940. Osservazioni morfologiche sul bacino di Colfiorito e presupposti idro-geologici della sua bonifica. Istituto geografico militare, p. 27.

Lotti B., 1917. L'antico Lago Tiberino e le ligniti dell'Umbria. Miniera Ital., 1, 274-275.

Malicky H., 2004. Atlas of European Trichoptera (2nd edn), Springer, Dordrecht, The Netherlands, $235 \mathrm{p}$.

Montanucci B. 1994-95. La tricotterofauna del tratto medio-alto del F. Topino (Umbria: PG). Scienze Naturali (Relatore: F. Cianficconi).

Moretti G.P., 1949. Contributo alla conoscenza della fauna delle Fonti del Clitunno (Foligno). Verh. Int. Verein. Limnol., 10, 344-352.

Moretti G.P., 1950. Tricotteri fonticoli dell'Appennino Umbro Marchigiano. Boll. Soc. Eustachiana, 42, 203-208.

Moretti G.P., 1952. Bilancio ecologico di una raccolta di Tricotteri delle Marche, Umbria ed Abruzzo. Studi sui Tricotteri XXII. Boll. Zool., 19, 245-269.

Moretti G.P., Cianficconi F., Salerno P. and Tucciarelli F., 1996. La Tricotterofauna dell'Umbria: considerazioni ecologiche e zoogeografiche. Boll. Mus. civ. St. nat.Verona, 20, 61-73.

Morroni M.P., 1948-49. Dati ecologici di un ochhio di fonte. Scienze Naturali (Relatore: G.P. Moretti).

Pantanelli D., 1886. Vertebrati fossili nelle ligniti di Spoleto. Atti della Società Toscana di Scienze Naturali, 7, 1.
Penna Rocchigiani M., 1949-50. Due anni di ricerche idrobiologiche alle sorgenti del F. Topino (Nocera Umbra). Scienze Naturali (Relatore: G.P. Moretti).

Pietrangeli C., 1953. Mevania (Bevagna), Istituto Nazionale di Studi Romani (Ed.), serie I, 13, Roma, p. 179.

Ravagli B., 1952-53. La biologia di un fontanile della piana umbra Scienze Naturali (Relatore: G.P. Moretti).

Regione Umbria, 2005. Progetto di cartografia geologica e geotematica a scala 1:10000: "Individuazione delle aree suscettibili di amplificazioni sismiche locali sui territori comunali con elevata pericolosità sismica di base".

Ricciolini C. 2000-01. Biologia ed ecologia della tricotterofauna di un corso d'acqua con substrato calcareo (F. Menotre). Scienze Biologiche (Relatore: C. Corallini).

Romozzi P. 1989-90. Aggiornamento della fauna tricotterologica delle sorgenti del M. Subasio. Scienze Biologiche (Relatore: F. Cianficconi).

Scaramucci A., 1948-49. La vita nelle sorgenti del F.Topino, Acquacci, Nocera Umbra (Artropodi e Molluschi). Scienze Naturali (Relatore: G.P. Moretti).

Sigismondi G., 1956. L'acqua Angelica e i Bagni di Nocera Umbra. Appunti storici. Coeditori Felice Bisleri \& C. e Raggruppamento Editoriale Medico Italiano, p. 79.

Todini B., 2005. La fauna macrobentonica per una moderna valutazione dei corsi d'acqua umbri. Dottorato di ricerca in: Biologia ed Ecologia, XVII ciclo: 162 p.

Todini B., Cianficconi F. and Corallini C., 2009. Biocenosi a Tricotteri nel Bacino del Topino (Umbria). Proceedings XXII Congr. Naz. Ital. Entomol. Ancona 15-18 Giugno.64.

Vigna Taglianti A., Audisio P. A., Belfiore C., Biondi M., Bologna M. A., Carpaneto G. M., De Biase A., De Felici S., Piattella E., Racheli T., Zapparoli M. and Zoia S., 1992. Riflessioni di gruppo sui corotipi fondamentali della fauna W-paleartica ed in particolare italiana. Biogeografia, 16, 159-179. 